\title{
OPEN Cardiomyocyte-specific deletion of $\beta$-catenin protects mouse hearts from ventricular arrhythmias after myocardial infarction
}

\author{
Jerry Wang ${ }^{1,2}$, Ying Xia ${ }^{1,2}$, Aizhu Lu ${ }^{1,2}$, Hongwei Wang ${ }^{1}$, Darryl R. Davis ${ }^{1,2}$, Peter Liu ${ }^{1,2}$, \\ Rob S. Beanlands ${ }^{1} \&$ Wenbin Liang ${ }^{1,2}$
}

Wnt/ $\beta$-catenin signaling is activated in the heart after myocardial infarction (MI). This study aims to investigate if $\beta$-catenin deletion affects post-MI ion channel gene alterations and ventricular tachycardias (VT). MI was induced by permanent ligation of left anterior descending artery in wildtype (WT) and cardiomyocyte-specific $\beta$-catenin knockout (KO) mice. KO mice showed reduced susceptibility to VT ( $18 \%$ vs. $77 \%$ in WT) at 8 weeks after MI, associated with reduced scar size and attenuated chamber dilation. qPCR analyses of both myocardial tissues and purified cardiomyocytes demonstrated upregulation of Wnt pathway genes in border and infarct regions after MI, including Wnt ligands (such as Wnt4) and receptors (such as Fzd1 and Fzd2). At 1 week after MI, cardiac sodium channel gene (Scn5a) transcript was reduced in WT but not in KO hearts, consistent with previous studies showing Scn5a inhibition by Wnt/ $\beta$-catenin signaling. At 8 weeks after MI when Wnt genes have declined, Scn5a returned to near sham levels and $\mathrm{K}^{+}$channel gene downregulations were not different between WT and KO mice. This study demonstrated that VT susceptibility in the chronic phase after $\mathrm{MI}$ is reduced in mice with cardiomyocyte-specific $\beta$-catenin deletion primarily through attenuated structural remodeling, but not ion channel gene alterations.

Each year, ventricular tachyarrhythmia-induced sudden cardiac death (SCD) claims the lives of millions of people worldwide, including $\sim 370,000$ Americans $^{1}$ and $~ 700,000$ European people ${ }^{2}$. Ischemic heart disease (myocardial infarction, MI) is the most common underlying disorder and accounts for $65-80 \%$ of the SCD cases ${ }^{3}$. In post-MI hearts, ventricular tachyarrhythmias (including tachycardia and fibrillation, VT/VF) ${ }^{4,5}$ result from both structural remodeling of the injured heart, i.e. fibrosis and scar formation in the infarcted region, and alterations in expression of ion channels/transporters especially in the border zone of the infarct ${ }^{6-8}$, such as reduced $\mathrm{Na}_{\mathrm{v}} 1.5^{9}$ and $\mathrm{Cx} 43^{10}$ as well as re-distribution of $\mathrm{Cx} 43$ (less in intercalated disk, but more in lateral surface of myocytes) ${ }^{10}$. The scar tissues are often interspersed with bundles of alive cardiomyocytes, which form "reentry circuit" together with border zone myocardium that has reduced and heterogenous conduction velocity providing the substrate for $\mathrm{VT} / \mathrm{VF}^{6}$. The alterations in ion channels in alive myocardium also increases their automaticity or triggered activity $^{11}$ that can initiate VT/VF.

The Wnt signaling is critical for embryonic cardiogenesis ${ }^{12}$ and we have recently shown that it regulates the differentiation of embryonic stem cells into different subtypes of cardiomyocytes ${ }^{13}$. In the canonical Wnt $/ \beta$ catenin pathway, the binding of Wnt ligands to their plasma membrane receptors leads to the cytoplasmic accumulation of $\beta$-catenin, followed by its translocation into the nucleus and activation or inhibition of the transcription of target genes. In healthy adult hearts, Wnt $\beta$-catenin signaling has a low activity but recent studies have suggested that its activity is increased in the myocardium in both human ischemic heart failure ${ }^{14}$ and animal models of myocardial infarction ${ }^{15}$ and heart failure ${ }^{16}$, as well as in murine models of cardiac hypertrophy induced by pressure overload or angiotensin $\mathrm{II}^{17-20}$. However, one study reported that increased Wnt $/ \beta$-catenin signaling was only found in the myocardium of post-MI pigs with hypercholesterolemia, but not in post-MI pigs with normal cholesterol levels ${ }^{21}$. Recent studies, including those from our group ${ }^{22-24}$, have demonstrated the capability of Wnt/ $\beta$-catenin signaling to regulate the expression of cardiac sodium channel gene $S c n 5 a$ (encoding $\left.\mathrm{Na}_{\mathrm{v}} 1.5\right)^{22-28}$. However, it remains unknown if Wnt/ $\beta$-catenin signaling plays a role in the post-MI alterations in cardiac ion channel genes. 
A

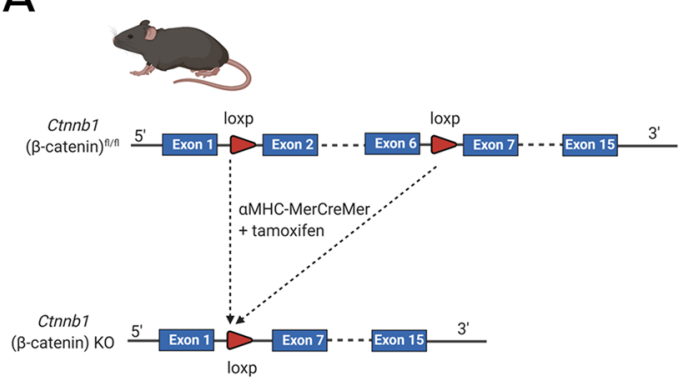

C

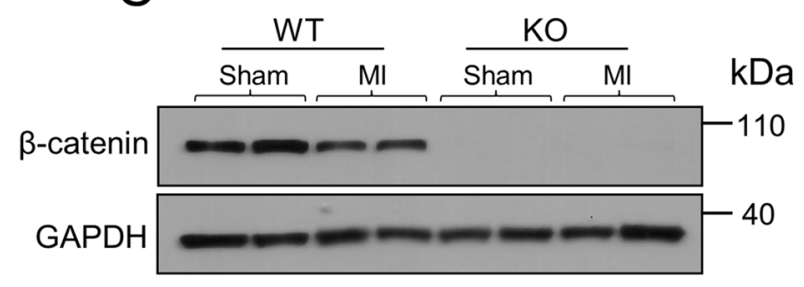

$E$ Mice at 1 or 8 weeks post LAD ligation

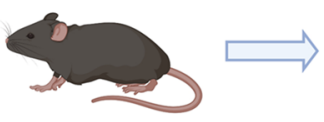

B

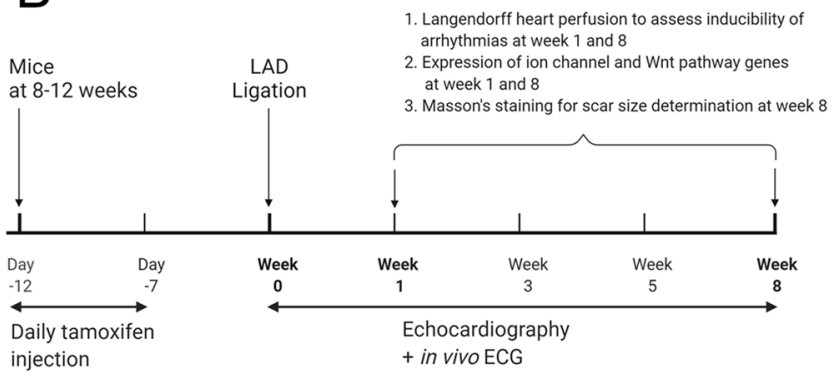

D

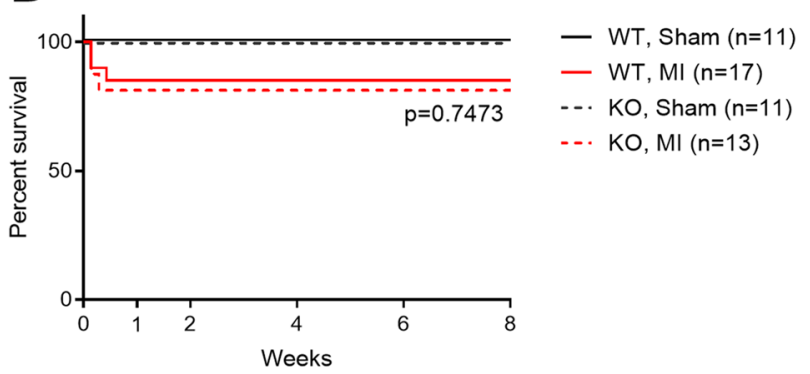

$\mathrm{F}$

Langendorff-perfused heart

+ programmed electrical stimulation (PES)

+ ex vivo ECG recording

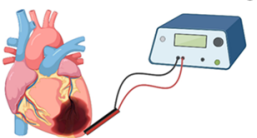

Programmed electrical stimulation

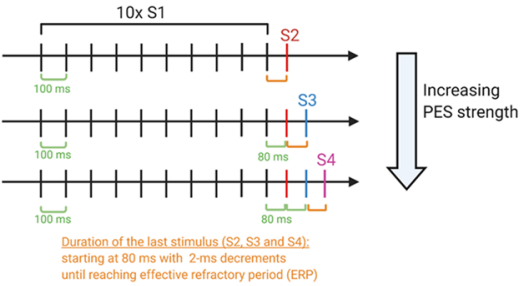

WT, MI (week 8)

KO, MI (Week 8)

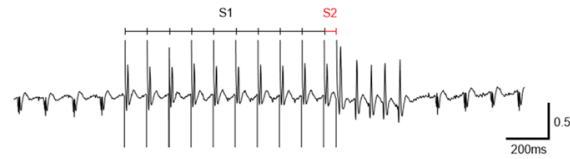

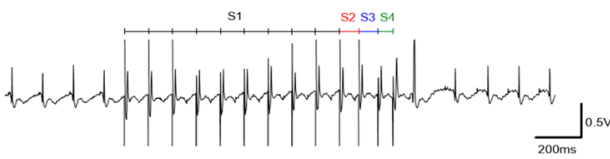

G

\begin{tabular}{|rc|}
\hline Arrhythmia & Score \\
\hline $\begin{array}{r}\text { VT (3 or more } \\
\text { consecutive PVCs) }\end{array}$ & 4 \\
\hline Couplet & 3 \\
\hline Bigemini & 2 \\
\hline Isolated PVCs & 1 \\
\hline $\begin{array}{r}\text { No arrythmias at } \\
\text { maximum PES } \\
\text { strength }\end{array}$ & 0 \\
\hline
\end{tabular}

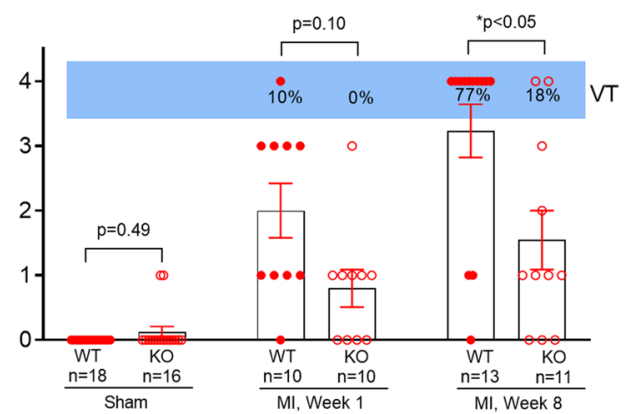


4Figure 1. $\beta$-catenin knockout reduces the susceptibility to ventricular tachycardias after myocardial infarction. (A) To generate cardiomyocyte-specific $\beta$-catenin (Ctnnb1) knockout mice, $C$ tnnb $1^{\text {flox/lox }}$ mice (with exons 2 to 6 floxed) were crossbred with aMHC-MerCreMer mice to obtain Ctnnb $1^{\text {flox/flox }}$;MHC-MerCreMer ${ }^{+/-}$mice, which were then treated with tamoxifen for deletion of exons 2-6 generating Ctnnb1 null allele due to the loss of the translational start site. Littermate Ctnnb1 $1^{\text {flox/flox }}$ aMHC-MerCreMer ${ }^{-/-}$mice were used as control wild-type mice. (B) Experimental design. (C) Representative western blot for confirmation of reduced total $\beta$-catenin in both sham and LAD-ligated (MI) KO hearts. Protein samples were prepared from left ventricular free wall of sham mice and from the border zone in mice at 8-weeks post-MI (myocardial infarction). GAPDH was used as a loading control. The original uncropped gel images are included in Supplementary Fig. 1. (D) Survival curve showing no difference in survival between WT and KO mice after LAD ligation ( $p=0.7473$, analyzed by Log-rank (Mantel-Cox) test). Only the mice in the long-term (8-weeks after MI) study were included in this survival analysis. All mortality occurred within the first week. (E) Protocols for evaluation of the inducibility of ventricular tachyarrhythmias (VT). Left Panel: Mouse hearts were isolated and Langendorff-perfused with Tyrode solution containing isoproterenol, ex vivo ECG were continuously measured by placing recording electrodes around the heart as we previously described ${ }^{23,54}$. Programmed electrical stimulation (PES) was applied using a MyoPacer (IonOptix) via a pair of platinum electrodes placed on the left ventricular apex of the heart. Right Panel: the standard stimulation protocol consisted of 10 stimuli at $100 \mathrm{~ms}$ intervals $(\mathrm{S} 1,5 \mathrm{~V})$ followed by one extra stimulus (S2) starting at an interval of $80 \mathrm{~ms}$ which was then reduced by $2 \mathrm{~ms}$ until the effective refractory period (ERP) was reached. If VT or VF was not induced, a second extra stimulus (S3) was added at $80 \mathrm{~ms}$ after S2. The S3 interval was then reduced by $2 \mathrm{~ms}$ until the ERP was reached. Finally, a third extra stimulus (S4) was added $80 \mathrm{~ms}$ after S3 and was then decreased by $2 \mathrm{~ms}$ until the ERP was reached. If a heart failed to develop a VT or VF with 3 extra stimuli, the heart was deemed non-inducible. (F) Representative ex vivo ECG (Lead II) showing PES-induced ventricular tachycardia (defined as 3 or more consecutive PVCs) in a WT heart (8-week after MI) when stimulated with one extra stimulus (S2), and only one single PVC in a KO heart (8-week after MI) when stimulated with three extra stimuluses (S4). (G) Summary of PES-induced PVCs and VTs in WT and KO hearts at 1 or 8 weeks after MI. An arrhythmia score was assigned to each heart according to the criteria shown in the left table. At 8 weeks after MI, VT was successfully induced in $77 \%$ of WT hearts but only in $18 \%$ of KO hearts. Data were analyzed by two-way ANOVA and Bonferroni post-hoc comparison.

On the other hand, previous studies have not reached a consensus regarding the role of Wnt/ $\beta$-catenin signaling in the structural remodeling of the post-MI hearts. Several studies have suggested that the Wnt/ $\beta$-catenin signaling is cardioprotective and reduces scar size. In post-MI rats, viral expression of a constitutively active form of $\beta$-catenin in the border zone reduced infarct size, which is associated with reduced apoptosis and increased cell proliferation in both cardiomyocytes and fibroblasts ${ }^{29}$. In mice with deletion of Lrp5, the co-receptor required for $\mathrm{Wnt} / \beta$-catenin pathway activation, the infarct size after MI was larger than in wild-type mice ${ }^{21}$. In mice with fibroblast-specific deletion of $\beta$-catenin, ischemia/reperfusion injury induced accelerated LV dilation and pump dysfunction ${ }^{15}$. By contrast, other studies have suggested a detrimental role of Wnt $/ \beta$-catenin signaling. In postMI mice, enhancement of the Wnt/ $\beta$-catenin signaling in myocardium via Wnt3a protein injection accentuated cardiac dysfunction by inhibition of cardiac progenitor cell proliferation and endogenous regeneration ${ }^{30}$. Mice overexpressing FrzA (Sfrp-1), an inhibitor of the Wnt signaling, had reduced scar size and cardiac rupture after MI, which are associated with reduced cell apoptosis in the scar region ${ }^{31}$. In mice with aMHC-Cre mediated $\beta$-catenin deletion, which made the cells non-responsive to Wnt stimulation, the scar size after MI was smaller which was associated with enhanced differentiation of a cardiac progenitor population (marked as $\alpha \mathrm{MHC}^{+}$/ $\mathrm{GATA}^{+} / \mathrm{Tbx}^{+}$, but $\mathrm{cTnT}^{-}$) into small $\mathrm{cTnT}^{+}$cardiomyocytes in the infarcted region ${ }^{32}$.

In the present study we demonstrated for the first time that mice with cardiomyocyte-specific deletion of $\beta$-catenin have reduced susceptibility to ventricular tachyarrhythmias at 8 weeks after MI, which is associated with attenuated structural remodeling.

\section{Results}

$\beta$-catenin knockout reduces the susceptibility to ventricular tachycardias after myocardial infarction. Successful knockout of $\beta$-catenin after tamoxifen treatment was confirmed by western blot showing marked reductions of $\beta$-catenin protein in the left ventricle of $C \operatorname{tnn} b 1^{\text {flox/flox }}$; $a \mathrm{MHC}-\mathrm{MerCreMer}^{+/-}(\mathrm{KO})$ mice as compared to Ctnnb $1^{\text {flox/flox }}$ aMHC-MerCreMer ${ }^{-/-}$(WT) mice (Fig. 1C). Post-MI survival curve showed that there was no difference $(p=0.75)$ in the death rate between WT and KO groups (Fig. 1D) with all the death $(15 \%$ for WT; $19 \%$ for KO) occurred within the first week in both groups. The absence of animal deaths in the late stage after MI suggests no lethal arrhythmias in these mice. This reflects a known limitation of studying arrhythmias in mice-the low rates of spontaneous arrhythmias due to their fast heart rate, short action potential duration and smaller heart size, as compared to human and large animal models (e.g., monkeys and pigs) of heart disease.

To investigate the susceptibility of mice to ventricular arrhythmias, we used a protocol that we recently developed for rodent hearts ${ }^{23,33}$ that combined adrenergic stimulation (with isoproterenol) and progressive programmed electrical stimulation (PES) in isolated, Langendorff-perfused hearts (Fig. 1E), while ex vivo ECG was recorded by placing electrodes around the heart. With this protocol, ventricular tachycardias (VT, defined as three or more consecutive ectopic ventricular beats) were successfully induced in 77\% (10/13) post-MI WT mice at week 8 , but in none $(0 / 18)$ of the sham-operated WT mice (Fig. 1F,G) validating our VT-inducing protocol. No difference was observed between male and female mice in VT inducibility (4/5 in male and 6/8 in female, $p=0.84)$ or arrhythmia score (Fig. $1 \mathrm{G})(3.40 \pm 0.60$ in male and $3.13 \pm 0.58$ in female, $p=0.76)$. In contrast, VT 
was induced in only $18 \%(2 / 11)$ of the post-MI KO mice at week 8 indicating reduced susceptibility to ventricular arrhythmias ( $p<0.05$ vs. WT, Fig. 1G,). Interestingly, a significant portion of the KO mice at week 8 after MI (55\% vs. $15 \%$ in WT) exhibited isolated or coupled premature ventricular contractions (PVCs) that did not develop into VT even when stimulated with the maximum PES strength (Fig. 1G). At week 1 after MI when the scar tissue was still immature, the majority of WT and KO hearts only exhibited isolated or coupled PVCs with VT induced in only one WT heart (Fig. 1G), suggesting a low inducibility of VT at this subacute phase.

$\beta$-catenin knockout reduces the prolongation of QRS duration and QT interval after myocardial infarction. Surface ECG recording in live animals showed no difference between sham-operated KO and WT mice in any of the parameters analyzed (QT interval, QRS duration, PR internal and RR internal, Fig. 2). WT mice that received LAD ligation (MI) surgery exhibited prolonged QT interval (starting at week 1) and increased QRS duration (starting at week 3), but showed no changes in PR or RR intervals, suggesting that MI led to left ventricular remodeling without significantly affecting the function of the central conduction system (sinoatrial node and atrioventricular node), although it is not clear if the intrinsic heart rate (i.e. after blocking the effects of autonomic nervous system) is altered. The MI-induced QT interval prolongation and QRS duration increases were attenuated in KO mice compared with WT mice (Fig. 2), which is consistent with reduced VT susceptibility in $\mathrm{KO}$ mice.

$\beta$-catenin knockout attenuates chamber dilation and pump dysfunction after myocardial infarction. Echocardiography (Fig. 3A) showed no difference between sham-operated KO and WT mice in LV chamber dimension (end diastolic volume, EDV, Fig. 3B) or pump function (ejection fraction, EF, Fig. 3C). Post-MI WT mice exhibited marked LV dilation (EDV $=118.0 \pm 16.5 \mu \mathrm{l}, \mathrm{n}=14 \mathrm{vs.} 42.0 \pm 2.5 \mu \mathrm{l}, \mathrm{n}=9$ in sham WT at week $8, p<0.01$, Fig. $3 \mathrm{~B})$ and pump dysfunction $(\mathrm{EF}=21.6 \pm 3.3 \%, \mathrm{n}=14$ vs. $64.7 \pm 1.5 \%, \mathrm{n}=9$ in sham WT at week $8, p<0.01$, Fig. 3C), but LV posterior wall thickness (LVPWd) was not changed ( $p=0.7488$ by ANOVA, Fig. $3 \mathrm{D}$ ). In post-MI KO mice, $\mathrm{LV}$ dilation (EDV $=77.8 \pm 7.8 \mu \mathrm{l}, \mathrm{n}=13$ vs. $44.4 \pm 3.1 \mu \mathrm{l}, \mathrm{n}=10$ in sham KO at 8 -week, $\mathrm{p}=0.064$, Fig. $3 \mathrm{~B})$ and pump dysfunction $(\mathrm{EF}=34.9 \pm 4.3 \%, \mathrm{n}=13$ vs. $65.3 \pm 1.1 \%, \mathrm{n}=10$ in sham KO at 8 -week, $\mathrm{p}<0.01$, Fig. $3 \mathrm{C})$ were attenuated $(p<0.01)$ compared with post-MI WT mice.

$\beta$-catenin knockout reduces scar sizes after myocardial infarction. Masson's trichrome staining of sham-operated hearts did not show any difference in gross morphology between KO and WT mice (Fig. 4A). Consistent with observations in echocardiogram, post-MI WT hearts showed LV chamber dilation and wall thinning (Fig. 4A). The scar size, as determined by the \% fibrotic area of the LV wall, was smaller in post-MI KO (Scar size $38.5 \pm 3.9 \%, \mathrm{n}=5$ vs. $50.3 \pm 3.1 \%, \mathrm{n}=6$ in post-MI WT at 8 -week, $p<0.05$, Fig. $4 \mathrm{~B}$ ).

Upregulation of Wnt pathway genes in myocardium after myocardial infarction. Wnt pathways are fine-tuned by the soluble Wnt ligands and Wnt inhibitors on the extracellular side of the plasma membrane, as well as the receptors and co-receptors on the plasma membrane (Fig. 5A). Our qPCR analyses of gene transcripts of the myocardial tissues, which contained both cardiomyocytes and other cell types such as fibroblasts (Fig. 5B), showed that a variety of Wnt ligands, inhibitors and receptors are upregulated in the border zone and infarct region of the mouse hearts after MI (Fig. 5 and 6).

Wnt ligands (Fig. 5C). Wnt1, a canonical Wnt ligand known to selectively activate the $\beta$-catenin pathway, was increased by 3-7 fold after MI ( $p<0.05$ in WT infarct at week 1 vs. WT sham; $p<0.05$ in KO infarct at week 8 vs. KO sham) and Wnt $3 a$, another canonical Wnt ligand, was increased by 2 fold $(p<0.05$ in WT border at week 1 vs. WT sham). Wnt7, a noncanonical Wnt ligand that selectively activate the $\beta$-catenin-independent pathways, was increased by $\sim 20$ fold in the infarct $(p<0.05$ in WT infarct at both week 1 and 8 and border at week 8 vs. WT sham, $p<0.05$ in KO infarct vs. KO sham). Six Wnt ligands (Wnt4, Wnt10a, Wnt2, Wnt5b, Wnt6 and Wnt9a) that have been reported to activate both $\beta$-catenin dependent and independent pathways or have less defined functions, were also increased after MI: Wnt 4 was increased by 30 fold in infarct at week $1(p<0.01$ in KO infarct vs. KO sham), Wnt10 increased by $\sim 5$ fold in infarct ( $p<0.05$ in KO infarct at week 1 and 8 vs. KO sham), Wnt 2 showed a trend of increase in infarct at week $8(p<0.05$ in KO infarct vs. KO sham), Wnt $5 b$ was increased by $2-3$ fold ( $p<0.01$ in WT border and infarct at week 1 , and $p<0.05$ in WT infarct at week 8 vs. WT sham), Wnt6 was increased by $49 \%$ in $\mathrm{KO}$ infarct at week 8 ( $p<0.05$ vs. KO sham), and $W n t 9 a$ was increased by $92 \%$ in $\mathrm{KO}$ infarct at week 8 ( $p<0.05$ vs. KO sham).

Wnt inhibitors (Fig. 5D). Secreted frizzled-related proteins (Sfrp) are soluble Wnt inhibitors that prevent the binding between Wnt ligands and their plasma membrane receptors (frizzled) ${ }^{34}$. At week 1 after MI, Sfrp2 showed the largest increase in both border and infarct (by 97-256 fold, $p<0.05$ vs. WT sham or KO sham), Sfrp3 also showed significant increases (by 16-32 fold, $p<0.05$ vs. WT sham or KO sham) and Sfrp1 was increased by $\sim 30$ fold in the infarct ( $p<0.05$ vs. WT sham or KO sham). At week 8 , all three Sfrp levels have declined in border zone but remained high in the infarct region ( $p<0.05$ vs. WT sham or KO sham). Dickkopf-related proteins (Dkk) are another group of soluble Wnt inhibitors that selectively inhibit Wnt/ $\beta$-catenin pathway by causing endocytosis of Wnt co-receptors (Lrp5/6) from the plasma membrane ${ }^{35}$. $D k k 3$ was increased by $6-11$ fold at week 1 ( $p<0.05$ vs. WT sham or KO sham) and was further increased to 22-32 fold in the infarct at week 8 ( $p<0.05$ vs. WT sham or KO sham). Dkk1 was marginally increased by $1.3-2.0$ fold $(p<0.05$ in KO border at week 1 vs. KO sham, $p<0.05$ in WT infarct at week 8 vs. WT sham), while $D k k 4$ was not affected by MI $(\mathrm{p}=0.801)$. 
A

\section{Sham MI (8-week)}
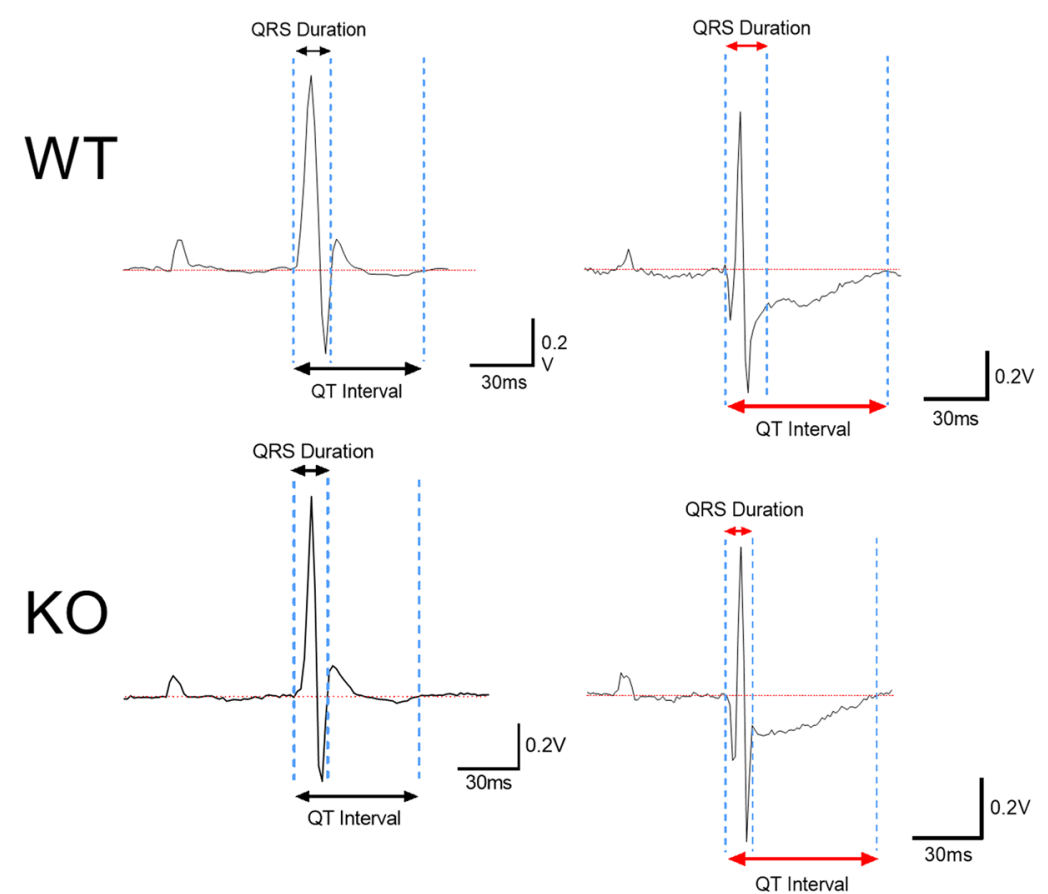

B
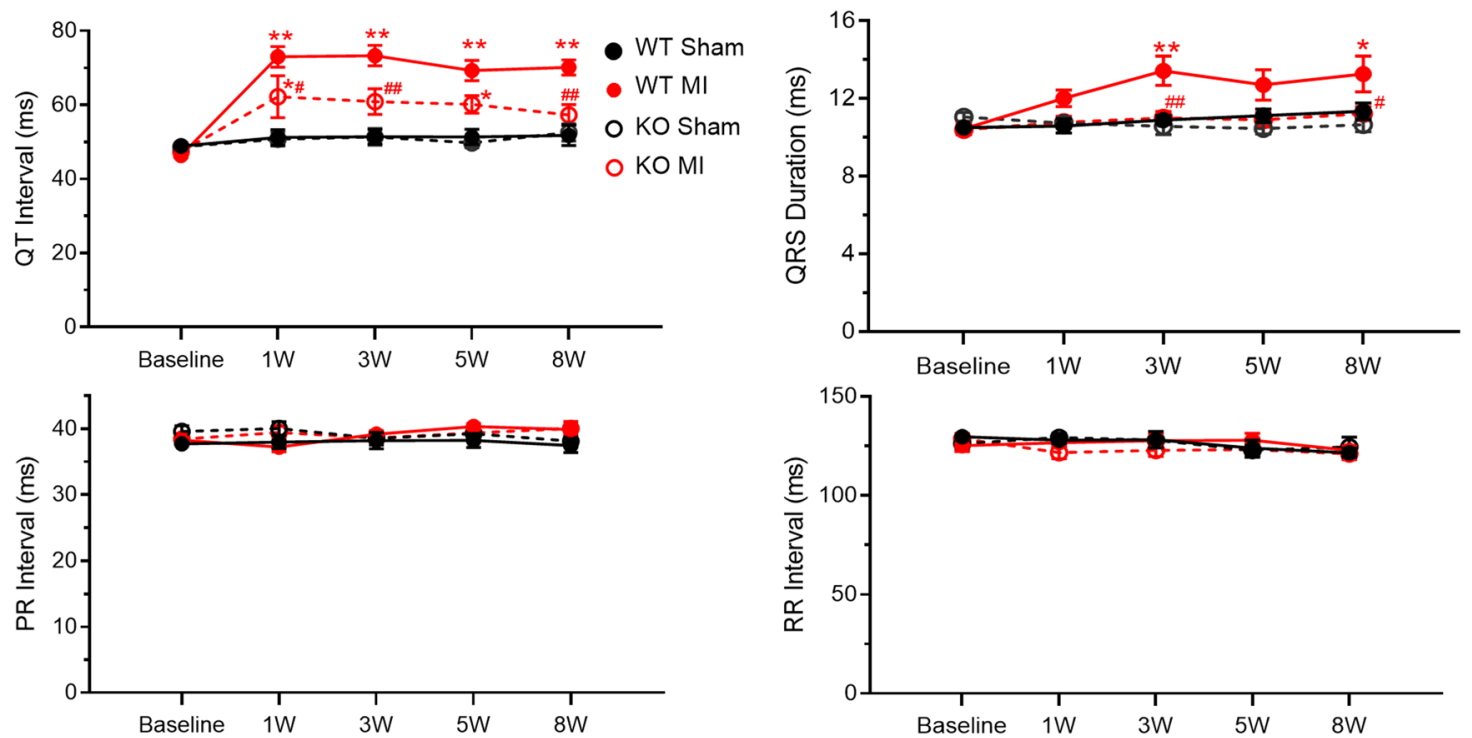

Figure 2. $\beta$-catenin knockout reduces the prolongation of QRS duration and QT interval after myocardial infarction. (A) Representative in vivo surface ECG traces (Lead II) in sham mice (left) and in mice at 8 weeks after MI (right). (B) Summary of in vivo ECG parameters, including QT Interval (top left), QRS duration (top right), PR Interval (bottom left), and RR Interval (bottom right). $\mathrm{n}=10-13$ per group. ${ }^{\star} p<0.05,{ }^{* *} p<0.01$, vs. corresponding sham groups; ${ }^{\#} p<0.05,{ }^{\# \#} p<0.01$, vs. WT MI group at the indicated timepoints. Data were analyzed by two-way ANOVA and Bonferroni post-hoc comparison.

Wht receptors and co-receptors (Fig. 6). Frizzled (Fzd) proteins are seven-transmembrane proteins that serve at the receptors for Wnt ligands on the plasma membrane (Fig. 5A). Among the $9 \mathrm{Fzd}$ genes, the mostly affected by MI were $F z d 1, F z d 2$ and $F z d 5$ that were increased in border and infarct at week 1 by $5-7$ fold ( $p<0.05$ vs. shams), $8-13$ fold ( $p<0.05$ vs. shams) and $2-4$ fold ( $p<0.05$ vs. shams), respectively. But in the border zones at week 8 they all showed a significant decline. $F z d 7, F z d 8$ and $F z d 3$ in infarct were increased at week 1 ( $p<0.05$ vs. shams), and $F z d 7$ and $F z d 8$ in infarct remained high at week 8 ( $p<0.05$ vs. shams). Fzd9 showed no changes at week 1 
A

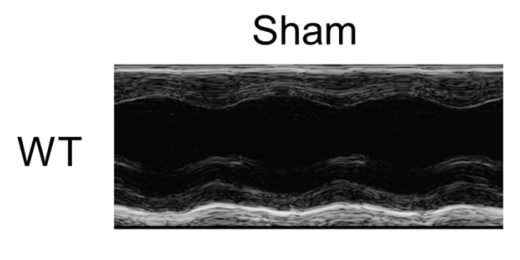

$\mathrm{KO}$

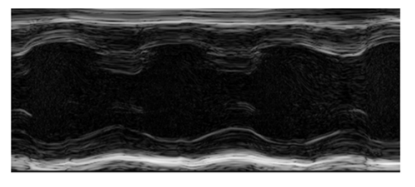

B

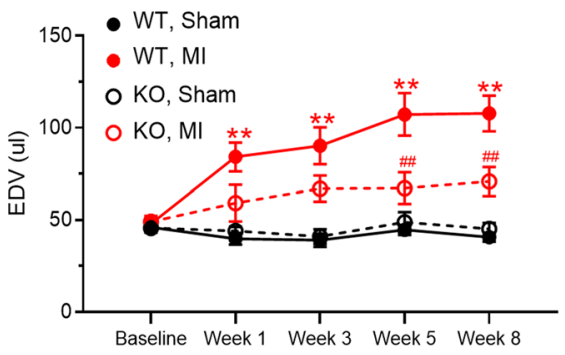

C

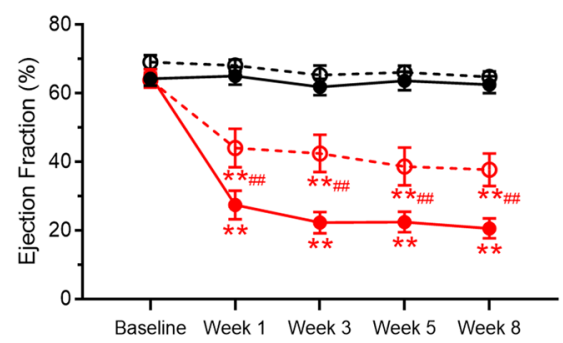

$\mathrm{D}$

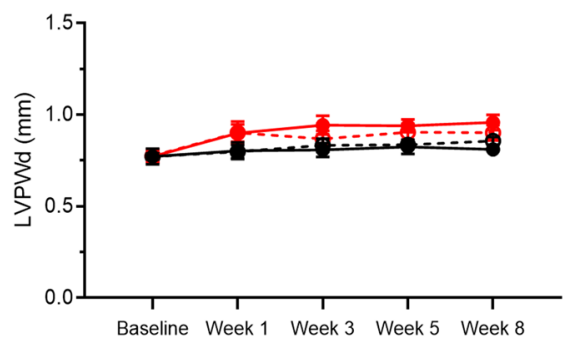

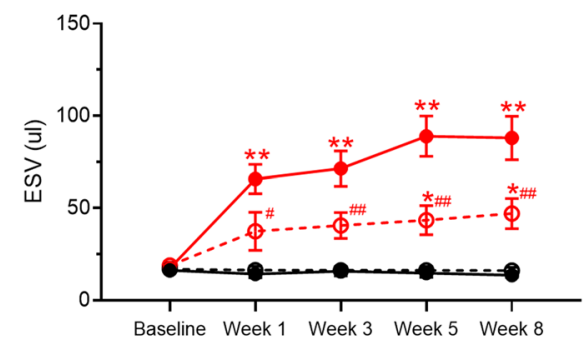

MI (8-week)
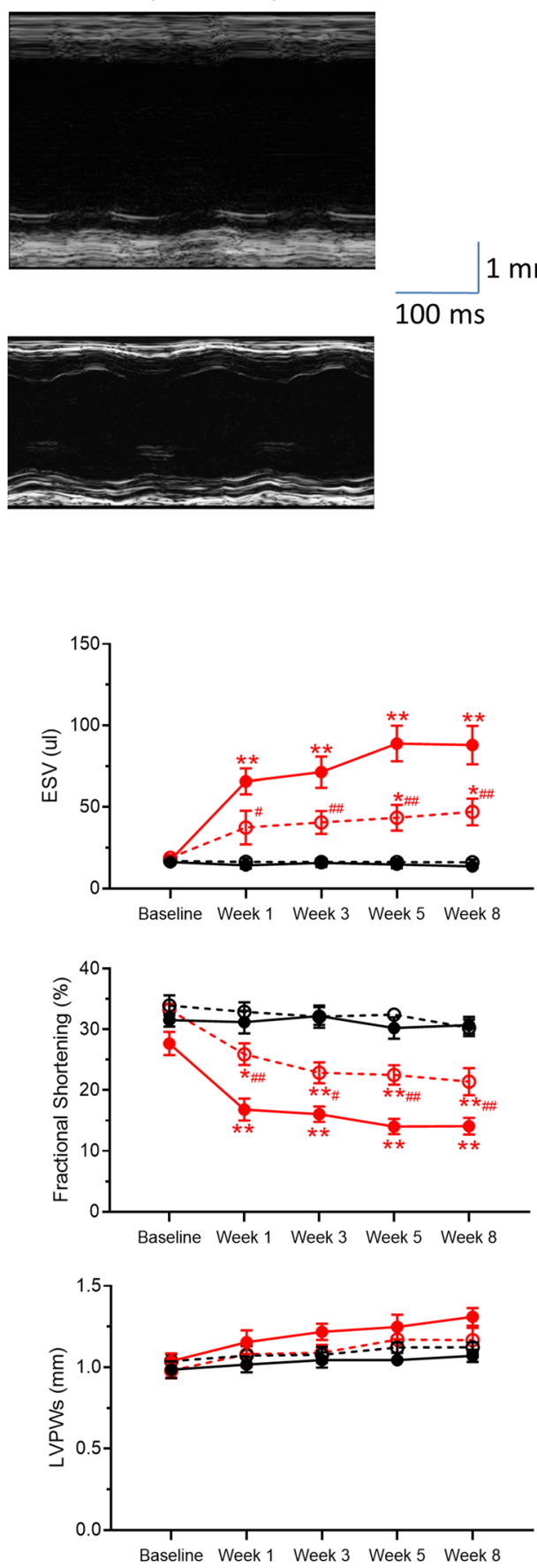

Figure 3. $\beta$-catenin knockout attenuates pump dysfunction and chamber dilation after myocardial infarction. (A) Representative short axis, M-mode echocardiographic recordings at the left ventricular mid-papillary level showing attenuation of cardiac function and dilation in $\beta$-catenin $\mathrm{KO}$ mice post-MI. Echocardiogram was recorded when heart rates were in the $400-500 \mathrm{bpm}$ range. (B) to (D) Summary of echocardiographic parameters of mice up to 8 weeks after MI showing reduced left ventricular dilation in $\mathrm{KO}$ mice as measured by end diastolic volume (EDV, panel B, left) and end systolic volume (ESV, panel B, right), attenuation of cardiac systolic dysfunction in $\mathrm{KO}$ mice as measured by ejection fraction (panel $\mathrm{C}$, left) and fractional shortening (panel C, right), and unchanged left ventricular posterior wall thickness at diastole (LVPWd, panel D, left) and at systole (LVPDs, panel D, right). $\mathrm{n}=10-13$ per group. ${ }^{\star} p<0.05,{ }^{\star *} p<0.01$, vs. corresponding sham groups; ${ }^{\#} p<0.05,{ }^{\# \#} p<0.01$, vs. WT MI group at the indicated timepoints. Data were analyzed by two-way ANOVA and Bonferroni post-hoc comparison. 
A

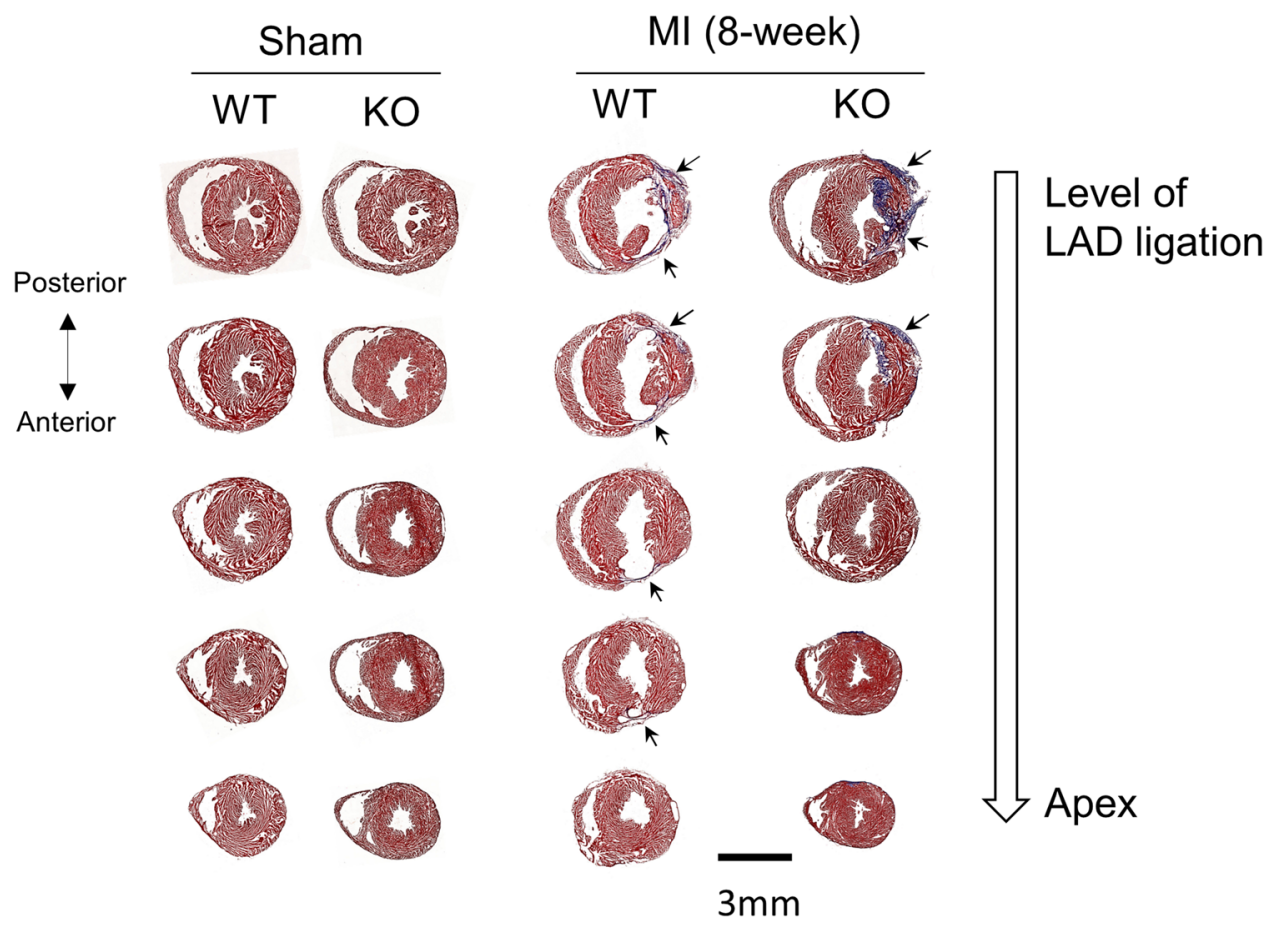

B

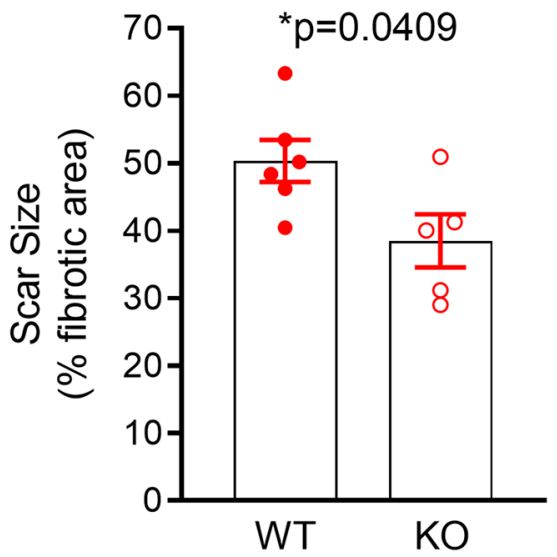

Figure 4. $\beta$-catenin knockout reduces scar sizes after myocardial infarction. (A) Representative images of Masson's trichrome staining of mouse heart tissue sections at 5 different levels from LAD ligation site (top) to the apex (bottom) at 8 weeks after MI (right), as well as in sham-operated hearts (left). The scar size for each the 5 different levels was calculated as the percentage of fibrotic area (as indicated by the arrows) to the total area of the left ventricle section. The heart's total scar size was then calculated as the mean of the scar sizes at the 5 levels and reported in panel B. (B) Summary of scar size showing reduced scar size in KO hearts $(n=5)$ compared to WT hearts $(n=6)$. Statistical analysis was performed with a two-tailed unpaired t-test.

but was increased at week 8 in infarct ( $p<0.05$ in WT infarct vs. WT sham). The other two Fzd genes in the family (Fzd4 and Fzd6) were not changed after MI $(p>0.20$ and $p>0.22$, respectively). Lipoprotein receptor-related protein 5 and $6(\mathrm{Lrp5} / 6)$ are co-receptors required for Wnt ligand-induced activation of $\beta$-catenin pathway (Fig. 5A). Lrp5 showed a 1.7-2.3 fold increase at week 1 ( $p<0.05$ in WT infarct and in KO border vs. shams). Lrp6 also showed a 1.7-2.3 fold increase at week 1 ( $p<0.05$ in WT infarct and in KO border vs. shams), and a $\sim$ twofold increase in infarct at week 8 ( $p<0.05$ in KO infarct vs. KO sham). But in the border zone at week 8 both Lrp5 and Lrp6 returned to near sham levels. Ror 2 and Vangl2 are co-receptors required for non-canonical Wnt mediated activation of $\beta$-catenin-independent pathways. Ror 2 and Vangl2 were increased in both border and infarct at week 1 by $4-9$ fold and $2-4$ fold ( $p<0.05$ vs. WT or KO shams), respectively, and they also showed a significant decline in border zone at week 8 . 


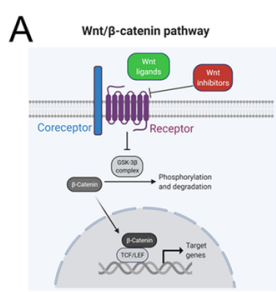

B

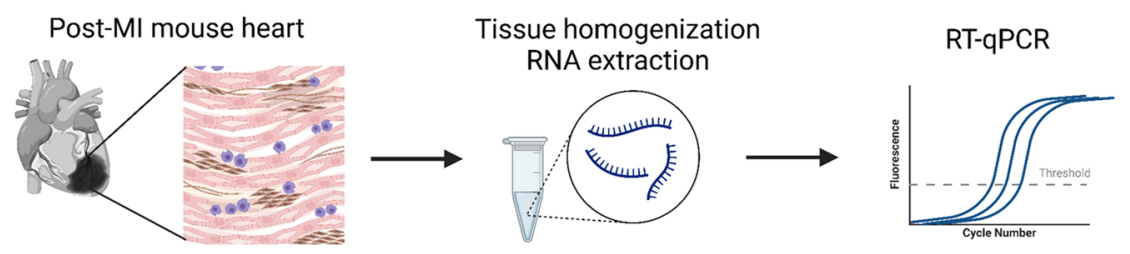

C Wnt ligands
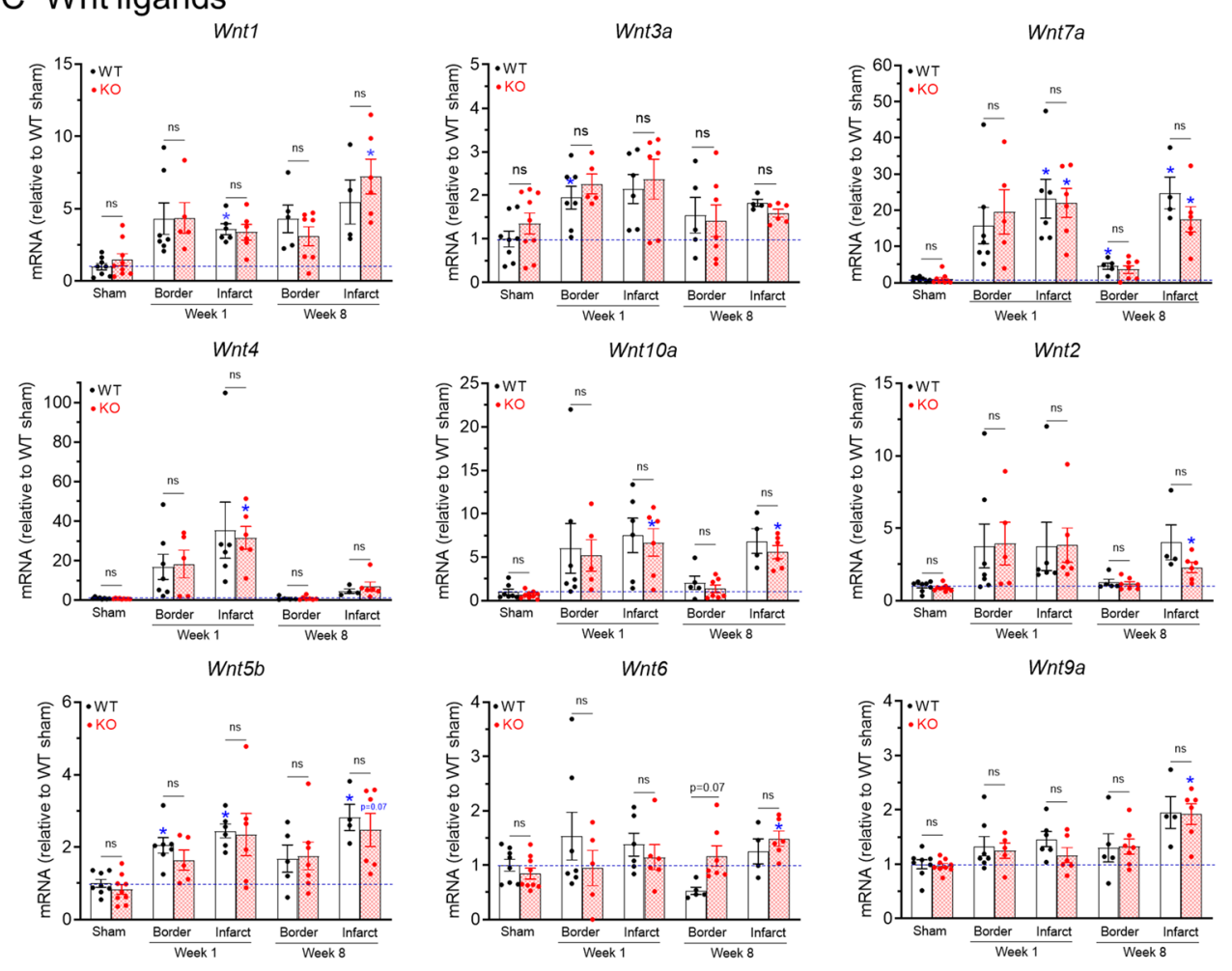

\section{Wnt inhibitors}
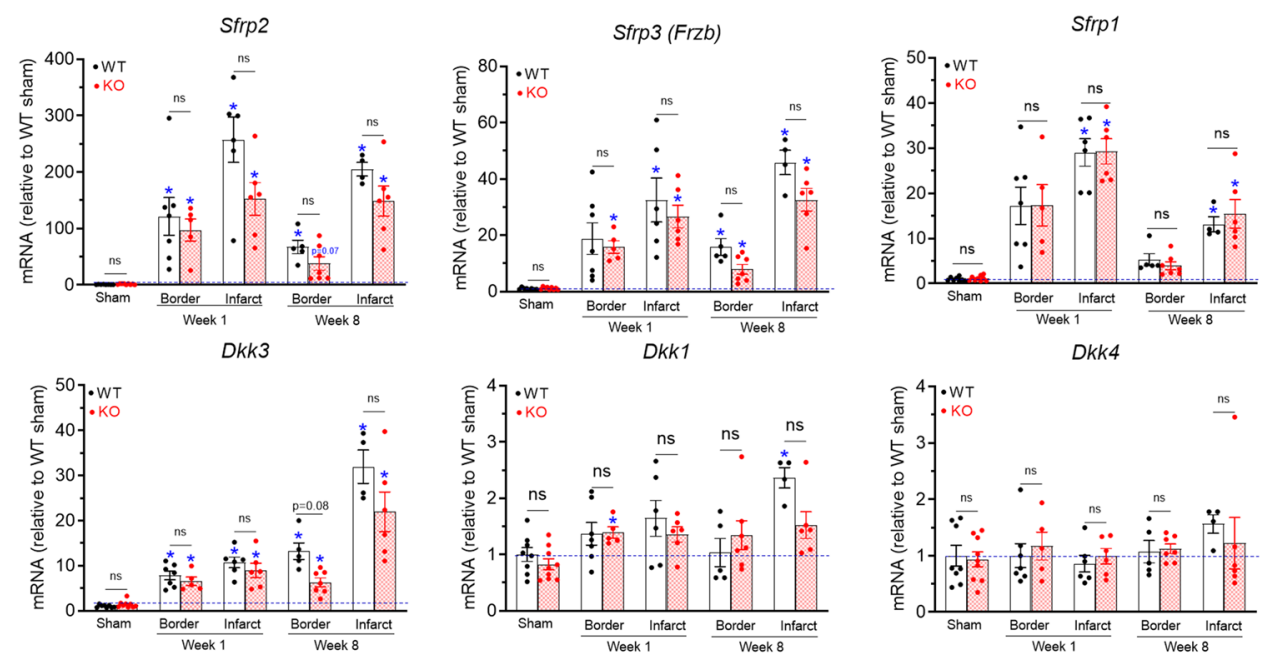
4Figure 5. Upregulation of Wnt agonists and antagonists in myocardial tissues after myocardial infarction. (A) Diagram of the canonical Wnt/ $\beta$-catenin signaling pathway, which is fine-tuned by the soluble Wnt ligands (green color) and Wnt inhibitors (red color) on the extracellular side of the plasma membrane. When a Wnt ligand binds to its plasma membrane receptor (purple color) and co-receptor (blue color), it leads to the inhibition of GSK-3 $\beta$ which is the key component of the $\beta$-catenin degradation complex; $\beta$-catenin will accumulate in the cytoplasm and then translocate into the nucleus where it, together with TCF/LEF, activate or inhibit the transcription of target genes. Abbreviations: GSK-3 $\beta$, glycogen synthase kinase $3 \beta$; TCF/LEF, T-cell factor/lymphoid enhancer factor. (B) Detection of gene transcript levels from RNA samples extracted from heart tissues, which contained different cell types, such as cardiomyocytes (pink color), fibroblasts (brown color) and inflammatory cells (blue color). (C) and (D) qRT-PCR analyses of transcript levels of Wnt ligands (C) and Wnt inhibitors (D) in sham, 1 -week post MI, and 8-week post MI infarct and border zone myocardium. $\mathrm{n}=4-10$ per group. ${ }^{\star} p<0.05$ vs. corresponding sham groups. "ns" means no significant difference between WT and KO groups. Data were analyzed by two-way ANOVA and Bonferroni post-hoc comparison.

$\beta$-catenin protein. In cells not stimulated with $\mathrm{Wnt} / \beta$-catenin pathway ligands, $\beta$-catenin in the cytoplasm is phosphorylated at Ser33/Ser37/Thr41 which labels them for ubiquitination and degradation ${ }^{36}$. The level of $\beta$-catenin that is not phosphorylated at Ser33/Ser37/Thr41 (i.e., active $\beta$-catenin) is an index of the Wnt/ $\beta$ catenin pathway activation in the cells ${ }^{37}$. As shown in Fig. $6 \mathrm{C}$, active $\beta$-catenin was increased by 4.9 fold in the border zone myocardium of WT hearts at 1 week after LAD ligation $(p<0.01$ vs. WT sham), suggesting activation of the Wnt/ $\beta$-catenin pathway in the myocardial tissue. An increase in active $\beta$-catenin at 1 week (3.9 fold, $p<0.05)$ was found in KO hearts in which only the cardiomyocytes were deleted of $\beta$-catenin. This suggests activation of the $\mathrm{Wnt} / \beta$-catenin pathway at 1 week in non-cardiomyocytes (such as fibroblasts and inflammatory cells). In addition, the increased total $\beta$-catenin in $\mathrm{KO}$ hearts at 1 week also suggests an increased proportion of non-cardiomyocytes (e.g., by fibroblast proliferation and infiltration of inflammatory cells) in the border zone myocardium.

Upregulation of Wnt pathway genes in ventricular myocytes after myocardial infarction. In order to investigate if Wnt pathway genes are altered in cardiomyocytes after MI, single cardiomyocytes were isolated from the infarct and border tissues (or from the left ventricular free wall in sham-operated groups) after collagenase digestion of the hearts (Fig. 7A). Cardiomyocytes were purified by removing non-cardiomyocyte cells after settlement for 10 min (Fig. 7A). qPCR analysis of pure cardiomyocytes showed that many of the Wnt pathway genes are also upregulated after MI (Fig. 7B to D).

Wht ligands (Fig. 7B). Wnt4 was increased in cardiomyocytes by $17-38$ fold at week 1 ( $p<0.05$ in KO vs. sham), which is similar to its increase in myocardial tissue $(\sim 30$ fold $)$. Wnt10 showed a trend of 8-13 fold increase in cardiomyocytes at week 1 ( $p=0.06$ in KO vs. sham). Wht 2 showed a trend of $4-6$ fold increase in cardiomyocytes at week 1 ( $p=0.06$ in both WT and KO vs. shams). Wnt1 was increased but to a lesser degree (by $\sim 30 \%, p<0.05)$ than in myocardial tissue (by 3-7 fold), while Wnt3a was not affected in cardiomyocytes $(p>0.25)$ after MI. Wnt7 was increased by $6-13$ fold ( $p<0.05$, WT week 1 vs. WT sham), which is less than its increase in myocardial tissue (20 fold).

Wnt inhibitors (Fig. 7C). Sfrp2 was increased by $52-68$ fold in cardiomyocytes at week $1(p<0.05$ in KO vs. sham) and by 16 fold at week 6 ( $p<0.05$ in KO vs. sham), which were less than its increase in myocardial tissues (97-256 fold at week 1). Sfrp1 and Sfrp3, which had 30 fold increases in myocardial tissues at week 1, were increased in cardiomyocytes by $8-17$ fold and $8-12$ fold, respectively $(p<0.05$ in both WT and KO vs. shams) but were not significantly affected at week $6 . D k k 3$ was increased by $7-13$ fold at week $1(p<0.05$ in both WT and $\mathrm{KO}$ vs. sham) which is similar to its increase in myocardial tissues (6-11 fold), while Dkk1 was not statistically affected $(p>0.09)$ after MI.

Wht receptors and co-receptors (Fig. 7D). Fzd2, which was increased by $8-13$ fold in myocardial tissues at week 1 , was increased in cardiomyocytes by $4-5$ fold at week 1 ( $p<0.05$ in both WT and KO vs. shams). Similarly, $F z d 1$, which was increased by $5-7$ fold in myocardial tissue, was increased in cardiomyocytes by $2-3$ fold at week 1 ( $p<0.05$ in KO vs. sham). Fzd7 was increased by $90 \%$ in KO at week 1 ( $p<0.05$ vs. sham). Fzd3, Fzd8 and Fzd9 were not statistically affected by MI $(p>0.57, p>0.09$ and $p>0.33$, respectively). Fzd5 was reduced by $40 \%$ in WT at week 1 ( $p<0.01$ vs. WT sham) and reduced by $54 \%$ in $\mathrm{KO}$ at week 6 ( $p<0.05$ vs. KO sham). Lrp5 showed a trend of increase in cardiomyocytes at week 1 but was not statistically significant ( $p=0.08$ in KO vs. sham), while $L r p 6$, which had a $\sim 2$ fold increase in myocardial tissue, was reduced in cardiomyocytes by $45 \%$ at week 1 ( $p>0.05$ in WT vs. sham). Ror 2 was increased (by 5-8 fold, $p<0.01$ ) to a similar level in myocardial tissue $(4-9$ fold) at week 1. Vangl2, which was increased by $2-4$ fold in myocardial tissue, was increased in cardiomyocytes only by $54 \%$ in $\mathrm{KO}$ at week 1 ( $p<0.05$ vs. KO sham).

Ion channel gene expressions after myocardial infarction. Ion channels genes for ventricular depolarizing currents (Fig. 8A). In the border zone at week 1, the cardiac $\mathrm{Na}^{+}$channel gene transcript $(\operatorname{Scn} 5 a$, encoding $\left.\mathrm{Na}_{\mathrm{v}} 1.5\right)$ was reduced in WT hearts $(0.544 \pm 0.07, \mathrm{n}=7$ vs. WT sham $1.0 \pm 0.07, \mathrm{n}=8, p<0.01)$, but it was not reduced in $\beta$-catenin KO hearts $(0.76 \pm 0.15, \mathrm{n}=5$ vs. KO sham $0.90 \pm 0.04, \mathrm{n}=9, p>0.99)$. Because it is known that Wnt $/ \beta$-catenin signaling reduces Scn 5 a level in cardiomyocytes ${ }^{22,23,25}$ these observations suggest that the Wnt/ $\beta$-catenin pathway likely plays a role in $\operatorname{Scn} 5$ a downregulation in the border zone of post-MI hearts. 
A Wnt receptors (Frizzled, Fzd)
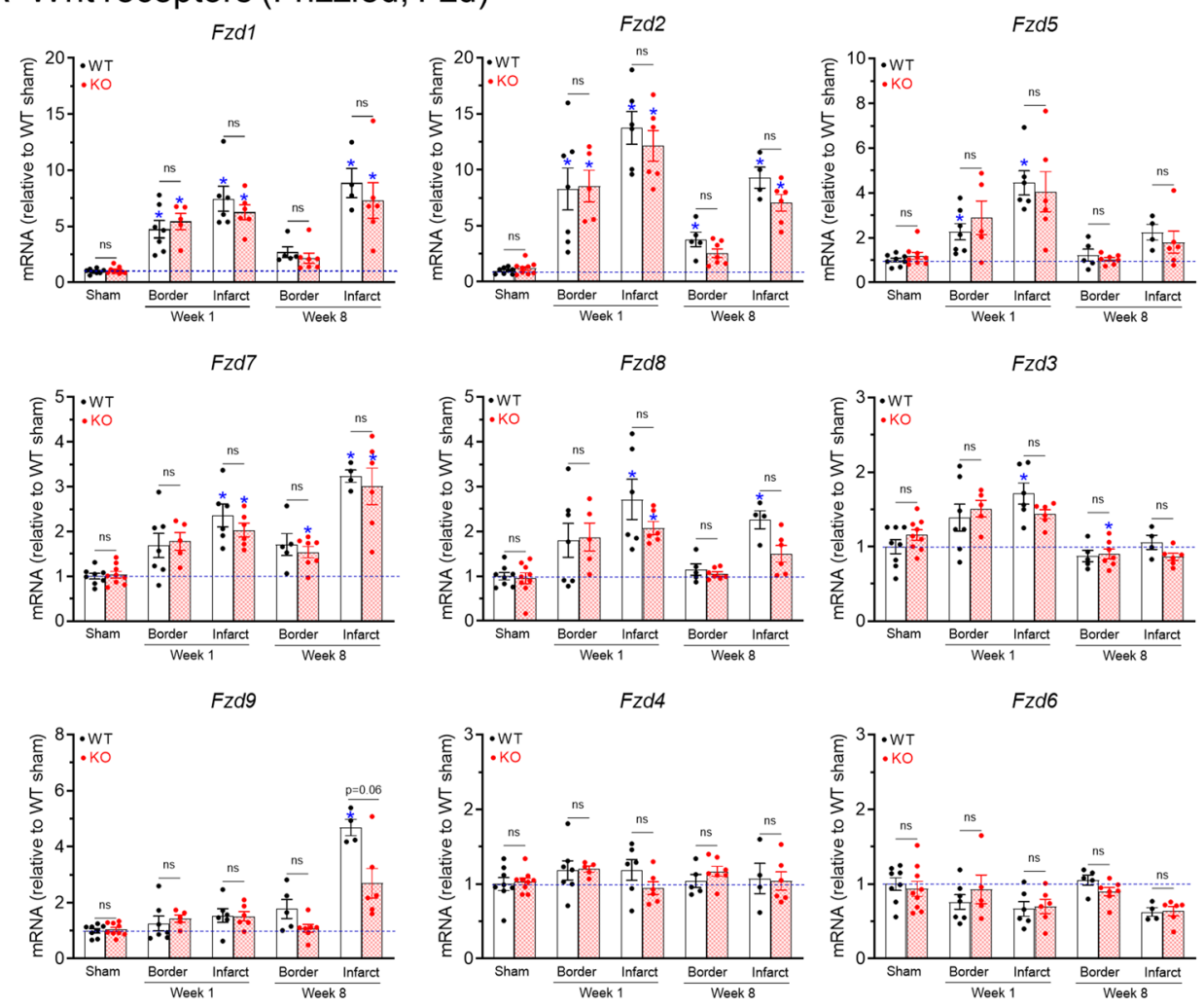

\section{B Wnt co-receptors}
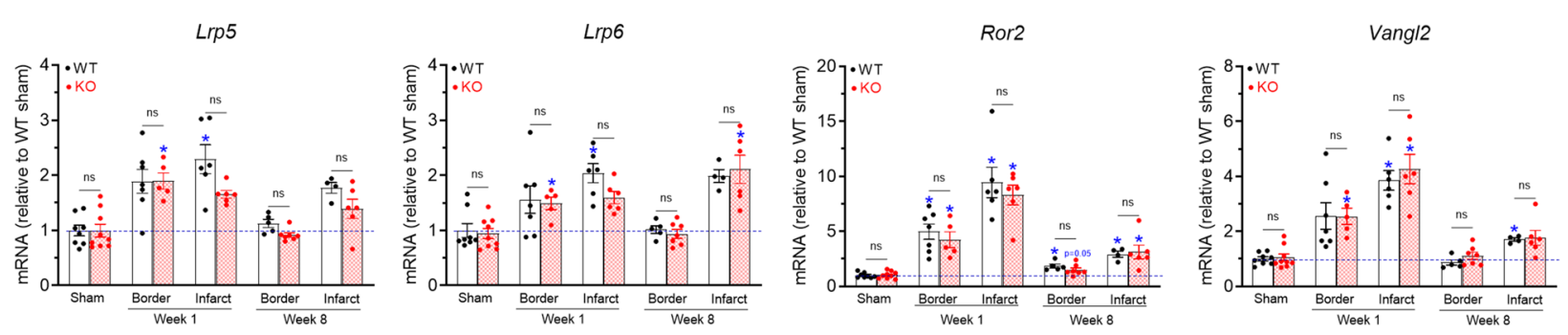

\section{C $\beta$-catenin protein}
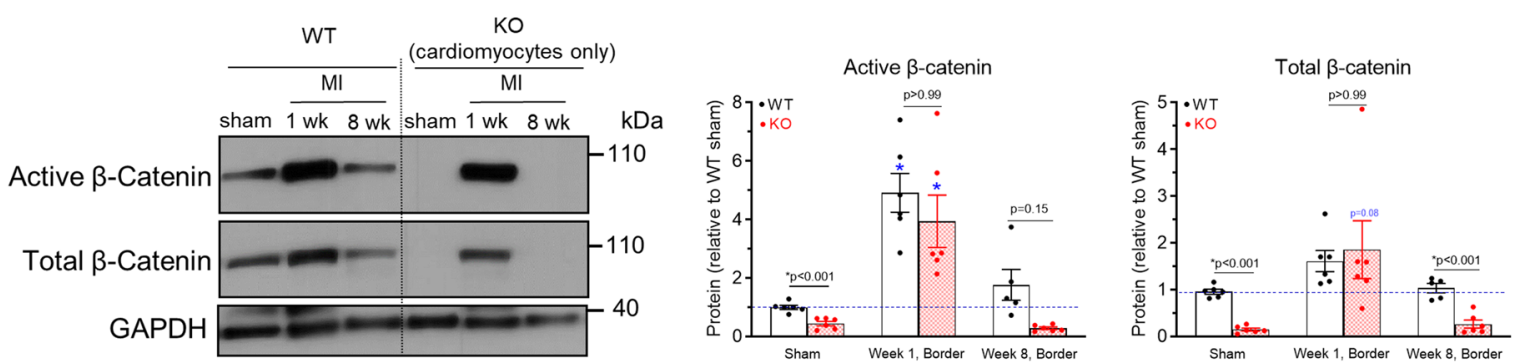

Figure 6. Upregulation of Wnt receptors and co-receptors in myocardial tissues after myocardial infarction. (A) and (B) qRT-PCR analyses of transcript levels of Wnt receptors (A) and Wnt co-receptors (B) in sham, 1-week post MI, and 8-week post MI infarct and border zone myocardium. $n=4-10$ per group. (C) Western blot of border zone tissue (MI, $n=5-6$ ) or left ventricular anterior free wall (sham, $n=5-6$ ) with anti-nonphospho (active) $\beta$-catenin (Ser33/Ser37/Thr41) antibody and anti-total $\beta$-catenin antibody. GAPDH was used as a loading control. The original uncropped gel images are included in Supplementary Fig. 1. ${ }^{\star} p<0.05$ vs. corresponding sham groups. "ns" means no significant difference between WT and KO groups. Data were analyzed by two-way ANOVA and Bonferroni post-hoc comparison. 


\section{A. Isolation of pure cardiomyocytes from mouse hearts}
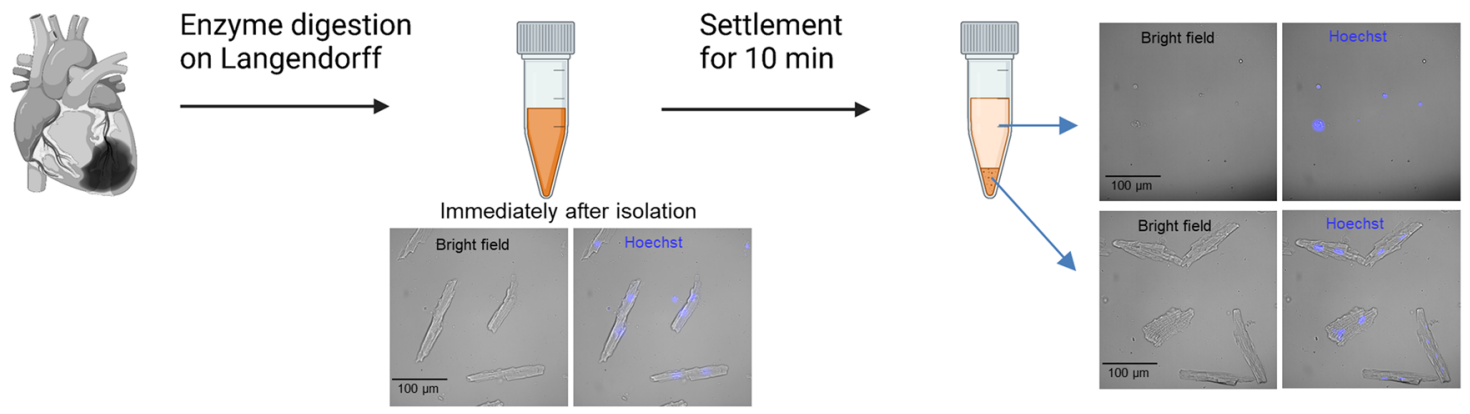

\section{B. Wnt ligands}

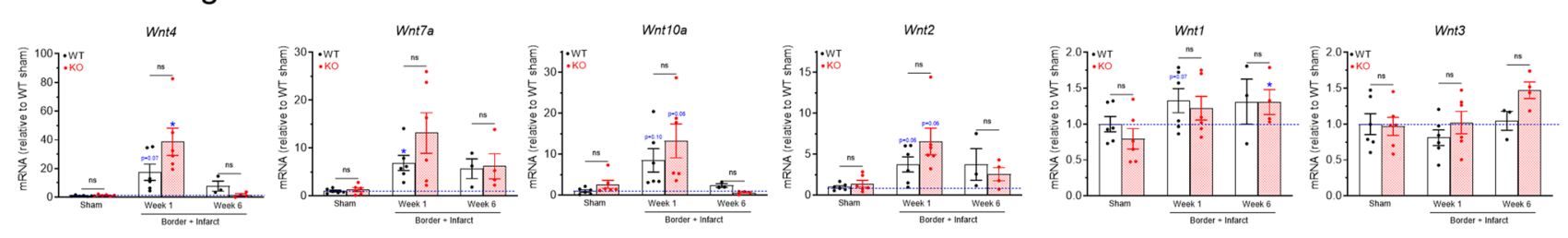

\section{Wnt inhibitors}
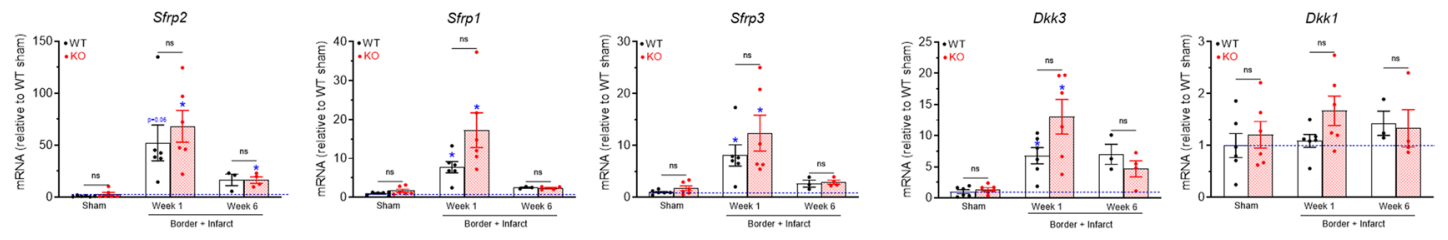

\section{Wnt receptors and co-receptors}
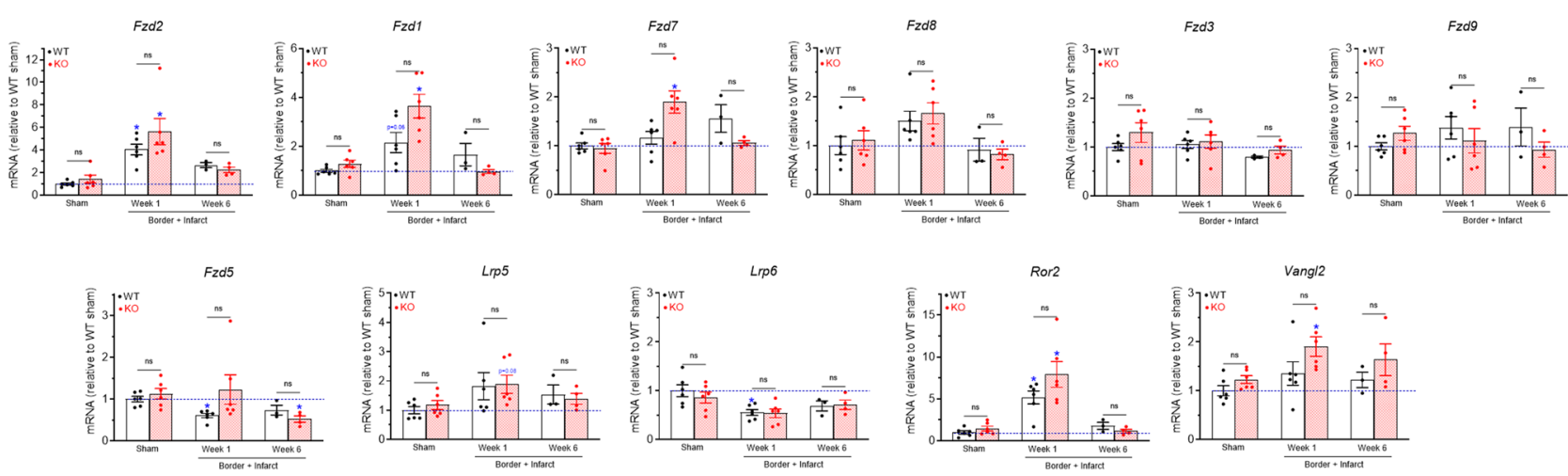

Figure 7. Upregulation of Wnt pathway genes in purified cardiomyocytes after myocardial infarction. (A) Isolation of pure cardiomyocytes from mouse hearts. Mouse hearts were digested with collagenase on a Langendorff perfusion system. The infarct and border region (or the left ventricular free wall in sham groups) were dissected, cut into small pieces, and gently pipetted to release single cells. The cell suspension contained both cardiomyocytes (rod-like cells) and other cell types (the Hoechst-positive small round cells), which can be separated by their different settlement speed: after a 10-min settlement, the cardiomyocytes were enriched in the pellet while the other cell types (non-cardiomyocytes) remained in the supernatant. The pellet containing pure cardiomyocytes was used for RNA extraction and qPCR analysis. The Hoechst-negative small particles are likely cell debris. (B) to (D) qRT-PCR analyses of transcript levels of Wnt ligands (B), Wnt inhibitors (C) and Wnt receptors and coreceptors genes (D) in pure cardiomyocytes isolated from sham, 1-week post MI, and 6-week post MI infarct and border zone. $\mathrm{n}=3-6$ per group. ${ }^{\star} p<0.05$ vs. corresponding sham groups. "ns" means no significant difference between WT and KO groups. Data were analyzed by two-way ANOVA and Bonferroni post-hoc comparison. 


\section{A. Ion channels important for ventricular action potentials}
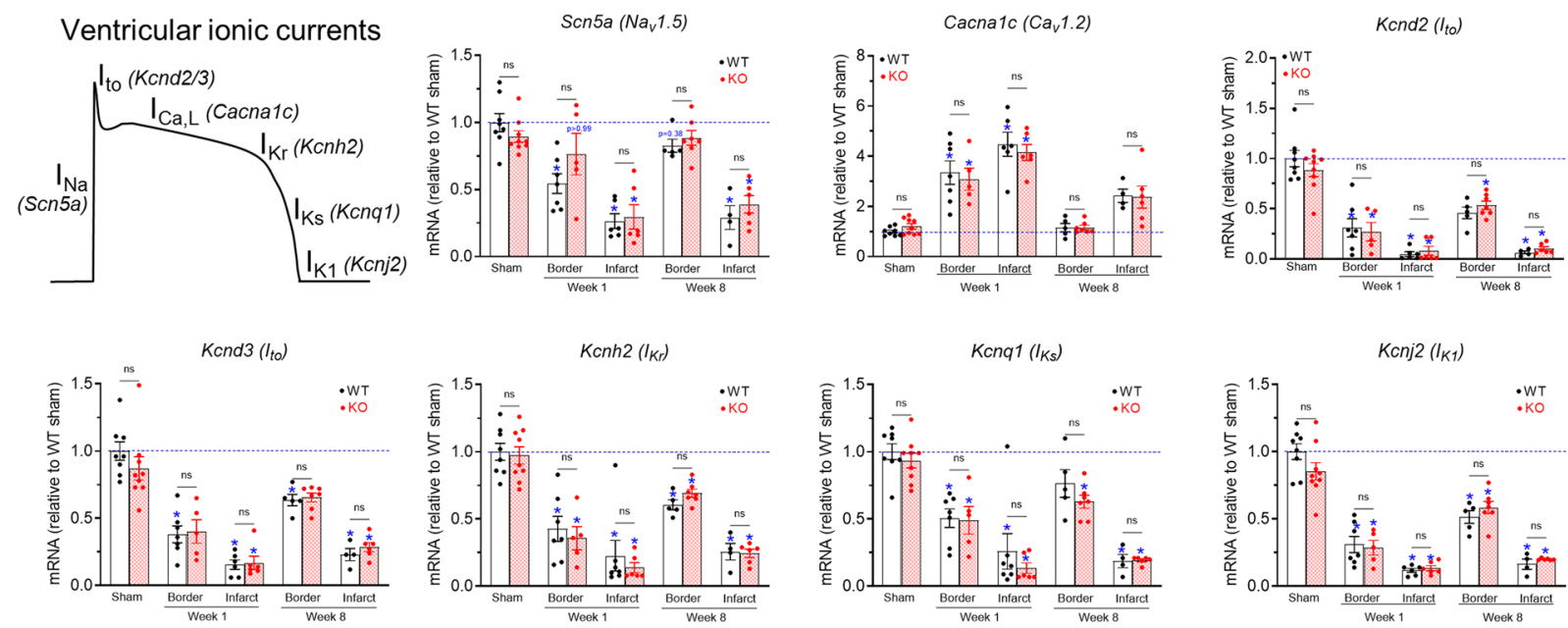

\section{B. Gap junctions and $\mathrm{HCN}$ channels}
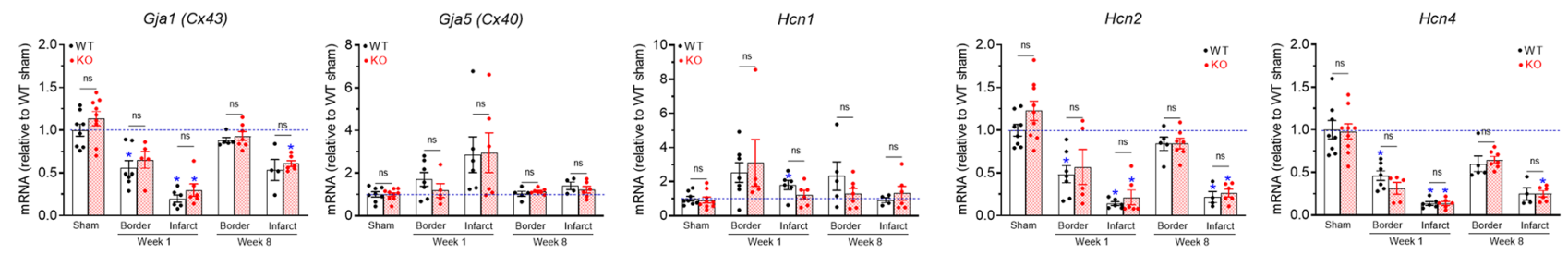

\section{Immunohistostaining of $\mathrm{Cx} 43$}

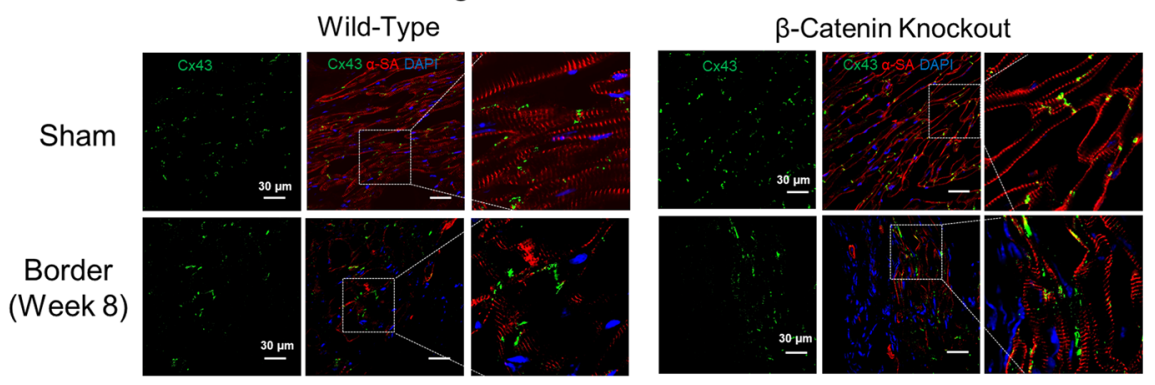

Figure 8. Ion channel gene expressions after myocardial infarction. (A) and (B) qRT-PCR analyses of transcript levels of ion channels involved in ventricular action potentials $(\mathbf{A})$, and gap junctions and $\mathrm{HCN}$ channels (B) in sham, 1-week post MI, and 8-week post MI infarct and border zone myocardium. $\mathrm{n}=4-10$ per group. ${ }^{\star} p<0.05$ vs. corresponding sham groups. "ns" means no significant difference between WT and KO groups. Data were analyzed by two-way ANOVA and Bonferroni post-hoc comparison. Abbreviations in panel A: $\mathrm{I}_{\mathrm{Na}}$, cardiac sodium current; $\mathrm{I}_{\mathrm{to}}$, transient outward potassium current; $\mathrm{I}_{\mathrm{Ca}, \mathrm{L}}$, L-type calcium current, $\mathrm{I}_{\mathrm{Kr}}$, rapidly activating delayed rectifier potassium current; $\mathrm{I}_{\mathrm{Ks}}$, slowly activating delayed rectifier potassium current; $\mathrm{I}_{\mathrm{K} 1}$, inwardly rectifying potassium current. (C) Representative confocal images showing similar distribution patterns of Connexin 43 (Cx43, green) in WT and KO myocardium. In sham hearts (top panels) of both WT and $\mathrm{KO}$ groups, $\mathrm{Cx} 43$ is restricted to the cell-cell junctions. In the border zone of hearts at 8-week after MI (bottom panels), both WT and KO groups showed a more diffusive pattern for Cx43. Cells were co-stained with $\alpha$-sarcomeric actinin ( $\alpha$-SA, red color, a marker of cardiomyocytes) and DAPI (blue).

In addition, the returning of $S c n 5 a$ level to near sham level in WT border at week $8(0.82 \pm 0.05, \mathrm{n}=5 \mathrm{vs}$. sham $1.0 \pm 0.07, \mathrm{n}=8, p=0.38$ ) also mirrored the reduced Wnt signaling at this time point. The significant reductions of $\operatorname{Scn} 5 a$ transcript in the infarct tissue at both week 1 and 8 are likely a result of the increased proportion of non-cardiomyocytes in this tissue. The L-type $\mathrm{Ca}^{2+}$ channel gene (Cacna1c, encoding $\left.\mathrm{Ca}_{\mathrm{v}} 1.2\right)$ was increased by 3.3 fold $(p<0.05)$ in both border and infarct tissues at week 1 as compared to sham groups. However, at week 8 
Cacna1c in the border zone has returned to near sham levels. The high level of Cacna1c in infarct may reflect the expression of this channel in fibroblast ${ }^{38}$ which are abundant in infarct tissues.

Ion channels genes for ventricular repolarizing currents (Fig. 8A). All the major ventricular $\mathrm{K}^{+}$channel genes, including $\mathrm{I}_{\text {to }}(K c n d 2, K c n d 3), \mathrm{I}_{\mathrm{Kr}}(K c n h 2), \mathrm{I}_{\mathrm{Ks}}(K c n q 1)$ and $\mathrm{I}_{\mathrm{K} 1}(K c n j 2)$, were reduced $(p<0.05)$ in border and infarct tissues at both week 1 and 8 as compared to sham groups. Consistent with our previous findings that Wnt signaling does not affect $\mathrm{I}_{\mathrm{K} 1}$ or action potential repolarization ${ }^{22}$, no differences were seen between KO and WT groups in these $\mathrm{K}^{+}$channel genes after MI.

Gap junctions and HCN channels (Fig. 8B). In the border zone, transcript of Cx43 (Gja1), the predominant gap junction isoform in ventricular myocardium, was reduced $(p<0.05)$ at week 1 but it returned to near sham levels at week 8 (Fig. 8B). However, immunostaining of $\mathrm{Cx} 43$ protein in border zones of both $\mathrm{KO}$ and WT hearts at week 8 showed that $\mathrm{Cx} 43$ is more diffusively distributed and is no longer restricted to cell-cell junction regions (intercalated disks) as seen in sham groups (Fig. 8C). Cx40 (Gja5), which is expressed in the Purkinje fibers, was not affected by MI. Among the genes of HCN channels that, if increased, will enhance myocyte automaticity, $H c n 1$ was increased while Hcn2 and Hcn4 were reduced at week 1 but they returned to near sham levels at week 8 (Fig. 8B). No differences were seen between KO and WT hearts in these gene transcript levels.

\section{Discussion}

Although Wnt/ $\beta$-catenin signaling has been studied in animal models of ischemic heart disease, this is the first study to investigate the post-MI arrhythmogenesis using $\beta$-catenin knockout mice. The reduced susceptibility to $\mathrm{VT}$ in $\beta$-catenin $\mathrm{KO}$ mice after MI is likely due to attenuated structural remodeling. The low VT inducibility at week-1 in both WT and KO mice when the scar was immature and at week- 8 in KO mice that had a smaller scar highlights the importance of structural remodeling in VT induction. Previous studies in patients have also demonstrated a positive relationship between scar sizes and the risk for $\mathrm{VT}^{39}$. In addition, large scar sizes are associated with a longer cycle length for monomorphic $\mathrm{VT}^{40}$, further supporting the reentry as the predominant mechanism for VT in post-MI hearts ${ }^{41}$.

The increased QRS duration, reflecting electrical dyssynchrony, is commonly found in heart failure patients and has been demonstrated to be an independent predictor of mortality in these patients ${ }^{42,43}$. Consistent with this, in the present study the increased QRS in post-MI WT hearts is associated with a greater VT inducibility. The increased QRS in these mice is likely secondary to the structural remodeling (scar and chamber dilation), as well as possible reductions in the conduction velocity of Purkinje fibers (arborization block) which is common in ischemic heart failure ${ }^{44}$. The QT interval and its dispersion are positively associated with the scar size in patients with $\mathrm{MI}$ and their increases are a risk factor for $\mathrm{VT}^{45}$. The attenuated increases in both QRS duration and QT intervals in $\beta$-catenin KO mice are consistent with their reduced VT inducibility and a smaller scar size.

The upregulation of Wnt pathway genes (such as Wnt1 and Wnt4) in the myocardial tissue of post-MI hearts is consistent with previous studies ${ }^{15,46}$. In addition, the present study provided direct evidence for the upregulation of Wnt genes in the cardiomyocytes. The similar levels of Wnt4 increase ( 30 fold $)$ in myocardial tissues and purified cardiomyocytes suggest that it is upregulated at the same degree in both cardiomyocytes and other cell types (such as fibroblasts, vascular cells and inflammatory cells). In contrast, other Wnt genes that are significantly increased in myocardial tissues are either upregulated at a lower level (such as Wnt1 and Wnt7) or not increased (such as Wnt3a) in cardiomyocytes, suggesting differential levels of upregulation in these Wnt genes between cardiomyocytes and other cell types. This finding may be helpful for future designing of therapeutic strategies for cell type-specific interventions of the Wnt pathways in ischemic heart disease.

While previous studies have reported conflicting results regarding the role of $\beta$-catenin in post-MI structural remodeling, our observations that $\beta$-catenin $\mathrm{KO}$ mice had attenuated structural remodeling after $\mathrm{MI}$ are consistent with the study by Zelarayan et al., which also found a smaller scar size in $\beta$-catenin KO mice at 4 weeks after MI (LAD ligation) ${ }^{32}$. The study by Zelarayan et al. also suggested that enhanced cardiac regeneration via activation of a cardiac progenitor population in the infarcted region may be a mechanism for the reduced scar in $\beta$-catenin $\mathrm{KO}$ hearts ${ }^{32}$. However, future studies are needed to investigate if other mechanisms, such as reduced cardiomyocyte deaths (in the form of necrosis, apoptosis, necroptosis and pyroptosis) or enhanced cardiomyocyte survival, are also involved. In addition to its role as the intracellular mediator of the Wnt/ $\beta$-catenin signaling, $\beta$-catenin is also expressed in the cytoplasmic side of the plasma membrane in the cell-cell junction region, where it interacts with other membrane proteins such as $\mathrm{N}$-cadherin ${ }^{47,48}$. Future studies are needed to investigate if the deletion of $\beta$-catenin in cardiomyocytes alters cell-cell interactions ${ }^{46}$ within the ischemic myocardium as a potential mechanism for the attenuated post-MI structural remodeling.

The reduced Scn $5 a$ transcript level in WT hearts, but not in KO hearts, at 1 week after MI is consistent with previous observations by us and others that $\mathrm{Wnt} / \beta$-catenin signaling reduces $S c n 5 a$ levels in cardiomyocytes ${ }^{22-28}$. Our finding that $S c n 5 a$ transcript returned to near sham levels in WT hearts at 8 weeks after MI is also consistent with the reduced Wnt gene levels at this timepoint as compared to 1 week. In addition, the similar levels of $\mathrm{K}^{+}$channel gene downregulations in both WT and KO hearts are consistent with our previous observation that Wnt $/ \beta$-catenin signaling does not affect the resting membrane potential or the repolarization phase of action potentials in rat cardiomyocytes ${ }^{22}$. HCN channels have been shown to play a role in the arrhythmogenesis of dilated cardiomyopathy, and HCN2-overexpressing hearts have increased VT susceptibility ${ }^{49}$. In the present study, the selective upregulation of $\mathrm{Hcn} 1$ gene is consistent with previous studies using a mouse model of cardiac hypertrophy, although $H c n 2$ and $H c n 4$ are more abundant than $H c n 1^{50}$. One limitation of the present study is that ion channel genes were not analyzed in purified cardiomyocytes and fibroblasts. Of note, the cardiac fibroblasts are known to express voltage-gated $\mathrm{K}^{+}$channels and transient receptor potential (TRP) channels ${ }^{51}$, and these cells 
can couple with cardiomyocytes in the myocardium to regulate electrical properties and arrhythmogenesis ${ }^{51}$. Future studies are needed to investigate the potential alterations in fibroblast ion channels and their contributions to the reduced arrhythmogenesis in $\beta$-catenin KO hearts after MI.

In summary, this study demonstrated that deletion of $\beta$-catenin reduces ventricular tachyarrhythmias in postMI hearts primarily through attenuated structural remodeling. However, this study only examined the ischemic heart failure mouse model, and future studies are warranted to investigate the Wnt/ $\beta$-catenin pathway activation in cardiomyocytes and its role in arrhythmogenesis in other types of heart failure, such as those induced by pressure overload or angiotensin $\mathrm{II}^{17-19}$.

\section{Methods}

All procedures were approved by the institutional animal care committee at the University of Ottawa (Protocol \#: HI-2602) and all methods were performed in accordance with the ARRIVE guidelines $2.0^{52}$. All methods were carried out in accordance with relevant guidelines and regulations.

Mice. To generate cardiomyocyte-specific $\beta$-catenin (Ctnnb1) knockout mice, $C$ tnnb $1^{\text {flox/llox }}$ mice (with exons 2 to 6 floxed, Jackson Lab, Stock No: 004152) were crossbred with aMHC-MerCreMer mice (Jackson Lab, Stock No: 005650) to obtain Ctnnb1 $1^{\text {flox/flox }}$; $a M H C-$ MerCreMer $^{+/-}$mice (Fig. 1A). Littermate Ctnnb $1^{\text {flox/flox }}$ aMHC$\mathrm{MerCreMer}^{-/-}$mice were used as control wild-type mice. At the age of $8-12$ weeks, all mice received daily subcutaneous injection of tamoxifen $(20 \mathrm{mg} / \mathrm{kg}$, Sigma, Catalogue No.:T5648, dissolved in sunflower seed oil at $10 \mathrm{mg} / \mathrm{ml}$ ) for 5 consecutive days (Fig. 1B). At 7 days after the last tamoxifen injection, baseline surface ECG and echocardiogram were recorded before the experimental myocardial infarction surgery as described below. Both male and female mice were used to investigate any sex-specific effects. A total of 51 male mice ( $26 \mathrm{WT}$ and 25 $\mathrm{KO}$ ) and 65 female mice ( $34 \mathrm{WT}$ and $31 \mathrm{KO}$ ) were used in this study.

Experimental myocardial infarction. Myocardial infarction was induced in mice by permanent ligation of the left anterior descending (LAD) coronary artery as we previously described ${ }^{53}$. Mice were injected with buprenorphine $(0.05 \mathrm{mg} / \mathrm{kg}$; subcutaneous) $1 \mathrm{~h}$ prior to surgery and twice daily thereafter for 3 days. During the surgery, mice were incubated, anesthetized using $2 \%$ isoflurane and maintained under physiologic temperature. The heart was exposed after a left thoracotomy, the LAD was identified under a dissection microscope (Topcon Corporation, Model No.: OMS-75) and ligated at $0.3 \mathrm{~mm}$ distal to the atrioventricular junction using a Prolene 7.0 suture. Successful LAD ligation was confirmed by the pale color of the affected myocardium before chest closure with a Prolene 6.0 suture.

Surface ECG. ECG with limb Lead I and II were recorded in mice at one day before and at week 1, 3, 5 and 8 after the LAD ligation using ADInstruments small animal ECG system (Powerlab 8/35 and Animal Bio Amp) and data were analyzed using LabChart 8.0. During ECG recording, mice were anesthetized with $1.5-2.0 \%$ isoflurane and body temperature was kept at $37^{\circ} \mathrm{C}$ using a heating pad. Mouse ECG parameters were analyzed in LabChart 8 software (version 8.1.5, ADInstruments) software. In brief, automated averaging of 10 consecutive beats was done by the software. Three consecutive "averaged cycles" were analyzed, and these values were finally averaged. To manually correct the auto-detected intervals if they were incorrectly placed: the PR interval was measured from onset of the P wave to the onset of the QRS complex (in cases where the Q wave was not very visible, the onset of the $\mathrm{R}$ wave was used). The QRS duration was measured from onset of the QRS complex to the return to baseline voltage following the $S$ wave (or if there was no return to baseline, the peak positive deflection following the $S$ wave was used). The QT interval was measured from onset of the QRS complex to the return to baseline voltage following the $\mathrm{T}$ wave.

Echocardiography. Echocardiography was recorded using a VEVO3100 system (VisualSonic Inc., Toronto) with the MS400 transducer (VisualSonic Inc., Toronto), and images were analyzed in VevoLab software (v.3.2.0 VisualSonic Inc., Toronto). During recording, animals were anaesthetized under $1.5-2 \%$ isoflurane with body temperature maintained at $37^{\circ} \mathrm{C}$ using a heating pad. To minimize the influence of heart rate on pump function, echocardiogram was recorded when heart rates were in the 400-500 bpm range. M-mode tracing of the left ventricle (LV) was recorded in the short-axis view at the mid-papillary level. Three consecutive cardiac cycles, measured in VevoLab, were averaged to determine ejection fraction \% (EF), fractional shortening \% (FS), end-diastolic volume (EDV), end-systolic volume (ESV), left ventricular internal diameter (LVIDs/LVIDd), and posterior wall thickness. The EF was calculated using the following equation: $\left(\mathrm{LVIDd}^{3}-\mathrm{LVIDs}^{3}\right) / \mathrm{LVIDd}^{3} \times 100$. FS was calculated using the following equation: (LVIDd-LVIDs)/LVIDd $\times 100$. B-mode tracing of the LV was recorded in the parasternal long-axis view and analyzed using the LV trace function in VevoLab to determine EF, FS, EDV, ESV, and LV volume.

Programmed electrical stimulation (PES) in isolated mouse hearts. At 1 and 8 weeks after MI, mouse hearts were isolated and Langendorff-perfused with Tyrode solution $\left(37^{\circ} \mathrm{C}\right)$ containing $1 \mu \mathrm{M}$ isoproterenol (Sigma, Catalogue No.: I6504), ex vivo ECG were continuously measured by placing recording electrodes around the heart as we previously described ${ }^{23,54}$ (Fig. 1E). PES was applied using a MyoPacer (IonOptix) via a pair of platinum electrodes placed on the left ventricular apex of the heart. The standard stimulation protocol (Fig. 1E) consisted of 10 stimuli at $100 \mathrm{~ms}$ intervals $(\mathrm{S} 1,5 \mathrm{~V})$ followed by one extra stimulus (S2) starting at an interval of $80 \mathrm{~ms}$ which was then reduced by $2 \mathrm{~ms}$ until the effective refractory period (ERP) was reached. If VT or VF was not induced, a second extra stimulus (S3) was added at $80 \mathrm{~ms}$ after S2. The S3 interval was then 
reduced by 2 ms until the ERP was reached. Finally, a third extra stimulus (S4) was added $80 \mathrm{~ms}$ after S3 and was then decreased by $2 \mathrm{~ms}$ until the ERP was reached. If a heart failed to develop a VT or VF with 3 extra stimuli, the heart was deemed non-inducible.

Masson's Trichrome staining. Mouse hearts were fixed by retrograde perfusion via the aorta with $4 \%$ paraformaldehyde (diluted in PBS) for $10 \mathrm{~min}$ at room temperature. The hearts were then incubated in increasing concentrations of sucrose dissolved in PBS (10\% for overnight, $20 \%$ for $8 \mathrm{~h}$, and $30 \%$ for overnight) on a horizontal rotator at $4{ }^{\circ} \mathrm{C}$ before being embedded in TissueTek OCT compound. Hearts were then cryosectioned at $10-\mu \mathrm{m}$ slices using a Leica vibrating microtome (Model No.: CM3050S) at five different levels of the heart from the ligation site to the apex at 300- $\mu \mathrm{m}$ intervals. Ten consecutive slices were made for each level. Heart sections were then stained using a Masson's Trichrome Staining kit (Sigma, Catalogue No.: HT15) according to the manufacturer's instructions. Fibrosis areas were analyzed with ImageJ software using the Colour Deconvolution for Masson's Trichrome Stain tool. The scar size for each the 5 different levels was calculated as the percentage of fibrotic area to the total area of the left ventricle section. The heart's total scar size was then calculated as the mean of the scar sizes at the 5 levels and reported in Fig. 4.

Immunohistostaining. Cryosectioned heart slices, as prepared above, were blocked and permeabilized in Dako protein block (Agilent, Catalogue No.: X0909) containing 0.1\% (w/v) saponin (Sigma, Catalogue No.: 47036) at room temperature for $90 \mathrm{~min}$. Slices were incubated with primary antibodies (see below) diluted in the same blocking solution at $4{ }^{\circ} \mathrm{C}$ for overnight. After washing with PBS, slices were incubated with secondary antibodies (see below) at room temperature for $1 \mathrm{~h}$. After washing with PBS for three times, slices were mounted with ProLong gold antifade reagent containing DAPI (ThermoFisher, Catalogue No.: P36931). Primary antibodies used were polycloncal rabbit anti-Cx43 (1:200, Sigma, Catalogue No.: C6219), and monoclonal mouse anti- $\alpha$-sarcomeric actinin (1:400, Sigma, Catalogue No.: A7811). Secondary antibodies used were anti-rabbit IgG (Alexa Fluor-488, 1:300) and anti-mouse IgG (Alexa Fluor-568, 1:300). Fluorescent images of the myocardium of left ventricular free wall of sham hearts and border zone of MI-hearts were taken with a ZEISS confocal microscope equipped with the Airyscan technique for high-resolution imaging (Zeiss Elyra S.1 LSM 880).

Isolation of single ventricular myocytes. Single ventricular myocytes were isolated from adult mouse hearts according to a protocol we previously described ${ }^{54-56}$. Mouse hearts were isolated and perfused on a Langendorff system with a nominally calcium-free Tyrode solution (in mmol/L: $136 \mathrm{NaCl}, 5.4 \mathrm{KCl}, 0.5 \mathrm{Na}_{2} \mathrm{HPO}_{4}, 10$ HEPES, $1 \mathrm{MgCl}_{2}$, and 10 glucose, $\mathrm{pH}=7.40$ with $\left.\mathrm{NaOH}\right)$ at $37^{\circ} \mathrm{C}$ for $10 \mathrm{~min}$. Collagenase $(1 \mathrm{mg} / \mathrm{ml}$, type II, Worthington Biochemical Inc. Catalogue No.:LS004177) and protease $(0.028 \mathrm{mg} / \mathrm{ml}$, type XIV, Sigma, Catalogue No.: P5147) were added into the calcium-free Tyrode solution and the hearts were perfused for another 15-20 min. The left ventricular tissue containing the infarct and border zone (in MI hearts) or the left ventricular free wall (in sham-operated hearts) was dissected and cut into small pieces in KB solution (in mmol/L: $100 \mathrm{~K}$-glutamate, $10 \mathrm{~K}$-aspartate, $2.5 \mathrm{KCl}, 10 \mathrm{KH}_{2} \mathrm{PO}_{4}, 2 \mathrm{MgSO}_{4}, 5 \mathrm{HEPES}, 20$ glucose, 20 taurine, 5 creatine, 0.5 EGTA, and $0.1 \%$ albumin, $\mathrm{pH}=7.2$ with $\mathrm{NaOH}$ ). Single cardiomyocytes were released in $\mathrm{KB}$ solution by gentle pipetting. Cell suspension was kept on ice and allowed to settle for $10 \mathrm{~min}$. A small aliquot $(50 \mu \mathrm{L})$ of the cells were stained with Hoechst 33,342 $(0.1 \mu \mathrm{g} / \mathrm{ml}$, ThermoFisher, Catalogue No.: 62249) for 10 min and cell images were taken using a fluorescent microscope (Leica Microsystems, Model No.: DMi8) equipped with a DAPI filter cube and a $40 \times$ objective. Cardiomyocytes settled to the bottom, while other cell types (such as fibroblasts, vascular endothelial and smooth muscle cells, and immune cells) remained in the supernatant (Fig. 7A). The cell pellet containing pure cardiomyocytes were used for RNA extraction and real-time PCR analysis.

Real-time quantitative PCR (qPCR). Total RNA was isolated from the border zone and infarct region of mouse hearts with Trizol reagent (ThermoFisher, Catalogue No.: 15596026) and digested with Turbo DNA-free kit (ThermoFisher, Catalogue No.: AM1907) to remove genomic DNA, according to manufacturer's instruction. Total RNA from purified ventricular myocytes was extracted using a RNeasy Micro kit (Qiagen, Catalogue No.:74004) and genomic DNA were removed by on-column digestion with RNase-Free DNase kit (Qiagen, Catalogue No.:79254) according to instructions of the manufacturer. One $\mu \mathrm{g}$ RNA was used for cDNA synthesis with a High-Capacity cDNA Reverse Transcription Kit (ThermoFisher, Catalogue No.: 4368814). Real-time quantitative PCR was performed on a CFX Connect Real-Time PCR Detection System (Bio-Rad) using standard SYBR green method. Information of the qPCR primers was included in Table S1 in the Online Supplement. No reverse transcriptase (RT-) controls and no template controls (NTC) were included for all the primers to make sure that there were no genomic DNA contamination or primer dimer signals. Transcript level of target genes was normalized to the level of Hprt 1 mRNA in the same sample. Results were analyzed with the $2^{-\Delta \Delta C(t)}$ method.

Western blotting. The border zone myocardial tissue of the post-LAD ligation hearts (week 1 and 8 ) or the left ventricular anterior free wall of sham-operated hearts were homogenized in T-PER Tissue Protein Extraction Reagent (ThermoFisher, 78510) containing Halt Protease Inhibitor Cocktail (ThermoFisher, 87786). Protein concentration was determined using Pierce Rapid Gold BCA Protein Assay Kit (ThermoFisher, A53226) and cell lysates ( $10 \mu \mathrm{g}$ protein per lane) were run on a $4-12 \%$ SDS-polyacrylamide gel and transferred onto a PVDF membrane. The transferred membrane was incubated with a primary antibody (see below) overnight at $4{ }^{\circ} \mathrm{C}$, followed by a 2 -h incubation with a peroxidase-conjugated secondary antibody (1:2000, Cell Signaling, \#7074). Primary antibodies used were rabbit anti-non-phospho (active) $\beta$-Catenin (Ser33/Ser37/Thr41) (1:1000, Cell signaling, \#8814) and rabbit anti-total $\beta$-Catenin (1:1000, Cell signaling, \#8480). Immunoreactivity was detected by chemiluminescence (Pierce ${ }^{\mathrm{Tm}}$ ECL Western Blotting Substrate, ThermoFisher, 32209). Equal protein loading 
of the gels was assessed by re-probing the membrane with rabbit anti-GAPDH antibody (1:2000, Cell Signaling, \#2118). Band densities were quantified using the "Gel Analyzer Protocol" function of ImageJ (https://imagej. nih.gov/ij/docs/menus/analyze.html\#gels), and presented as values after normalization to GAPDH in the same samples. All the original uncropped gel images are included in Supplementary Fig. 1.

Statistical analysis. Statistical analysis adhered to the Journal Guidelines. Data are expressed as mean \pm SEM with $p<0.05$ considered significant. Sample number indicates the number of biological replicates (each individual mouse is considered one sample). Differences between two means were evaluated by two-tailed Student's $t$-test. Differences among multiple means were assessed by one-way or two-way analysis of variance (ANOVA). When significance was detected by ANOVA, differences among individual means were evaluated post hoc by Bonferroni's test.

Received: 4 April 2021; Accepted: 23 August 2021

Published online: 06 September 2021

\section{References}

1. Benjamin, E. J. et al. Heart Disease and Stroke Statistics-2018 Update: A Report From the American Heart Association. Circulation 137, e67-e492. https://doi.org/10.1161/cir.0000000000000558 (2018).

2. Chugh, S. S. Sudden cardiac death in 2017: Spotlight on prediction and prevention. Int. J. Cardiol. 237, 2-5. https://doi.org/10. 1016/j.ijcard.2017.03.086 (2017).

3. Zheng, Z. J., Croft, J. B., Giles, W. H. \& Mensah, G. A. Sudden cardiac death in the United States, 1989 to 1998. Circulation 104, 2158-2163 (2001).

4. Roy, D. et al. Long-term reproducibility and significance of provokable ventricular arrhythmias after myocardial infarction. J. Am. Coll. Cardiol. 8, 32-39 (1986).

5. Marchlinski, F. E., Waxman, H. L., Buxton, A. E. \& Josephson, M. E. Sustained ventricular tachyarrhythmias during the early postinfarction period: electrophysiologic findings and prognosis for survival. J. Am. Coll. Cardiol. 2, 240-250 (1983).

6. Jackson, N., Gizurarson, S., Massé, S. \& Nanthakumar, K. in Cardiac Electrophysiology: From Cell to Bedside, 2018 (eds D. P. Zipes, J. Jalife, \& W. G. Stevenson) Ch. 48

7. Tomaselli, G. F. \& Zipes, D. P. What causes sudden death in heart failure?. Circ. Res. 95, 754-763. https://doi.org/10.1161/01.RES. 0000145047.14691.db (2004).

8. Tomaselli, G. F. \& Marbán, E. Electrophysiological remodeling in hypertrophy and heart failure. Cardiovasc. Res. 42, $270-283$. https://doi.org/10.1016/s0008-6363(99)00017-6 (1999).

9. $\mathrm{Pu}, \mathrm{J}$. \& Boyden, P. A. Alterations of $\mathrm{Na}+$ currents in myocytes from epicardial border zone of the infarcted heart. A possible ionic mechanism for reduced excitability and postrepolarization refractoriness. Circ. Res. 81, 110-119 (1997).

10. Wit, A. L. \& Peters, N. S. The role of gap junctions in the arrhythmias of ischemia and infarction. Heart Rhythm 9, 308-311. https:// doi.org/10.1016/j.hrthm.2011.09.056 (2012).

11. Gilmour, R. F. Jr., Heger, J. J., Prystowsky, E. N. \& Zipes, D. P. Cellular electrophysiologic abnormalities of diseased human ventricular myocardium. Am. J. Cardiol. 51, 137-144. https://doi.org/10.1016/s0002-9149(83)80024-1 (1983).

12. Gessert, S. \& Kühl, M. The multiple phases and faces of wnt signaling during cardiac differentiation and development. Circ. Res. 107, 186-199. https://doi.org/10.1161/circresaha.110.221531 (2010).

13. Liang, W. et al. Canonical Wnt signaling promotes pacemaker cell specification of cardiac mesodermal cells derived from mouse and human embryonic stem cells. Stem Cells 38, 352-368. https://doi.org/10.1002/stem.3106 (2020).

14. Hou, N. et al. Transcription factor 7-like 2 mediates canonical Wnt/beta-catenin signaling and c-Myc upregulation in heart failure. Circ. Heart Fail 9, e003010. https://doi.org/10.1161/circheartfailure.116.003010 (2016).

15. Duan, J. et al. Wnt1/betacatenin injury response activates the epicardium and cardiac fibroblasts to promote cardiac repair. Embo J. 31, 429-442. https://doi.org/10.1038/emboj.2011.418 (2012).

16. Dawson, K., Aflaki, M. \& Nattel, S. Role of the Wnt-Frizzled system in cardiac pathophysiology: A rapidly developing, poorly understood area with enormous potential. J. Physiol. 591, 1409-1432. https://doi.org/10.1113/jphysiol.2012.235382 (2013).

17. Iyer, L. M. et al. A context-specific cardiac $\beta$-catenin and GATA4 interaction influences TCF7L 2 occupancy and remodels chromatin driving disease progression in the adult heart. Nucleic Acids Res. 46, 2850-2867. https://doi.org/10.1093/nar/gky049 (2018).

18. Malekar, P. et al. Wnt signaling is critical for maladaptive cardiac hypertrophy and accelerates myocardial remodeling. Hypertension 55, 939-945. https://doi.org/10.1161/HYPERTENSIONAHA.109.141127 (2010).

19. Zhao, Y. et al. An essential role for Wnt/ $\beta$-catenin signaling in mediating hypertensive heart disease. Sci. Rep. 8, 8996. https://doi. org/10.1038/s41598-018-27064-2 (2018).

20. Bergmann, M. W. WNT signaling in adult cardiac hypertrophy and remodeling: Lessons learned from cardiac development. Circ. Res. 107, 1198-1208. https://doi.org/10.1161/circresaha.110.223768 (2010).

21. Borrell-Pages, M. et al. LRP5/canonical Wnt signalling and healing of ischemic myocardium. Basic Res. Cardiol. 111, 67. https:// doi.org/10.1007/s00395-016-0585-y (2016).

22. Liang, W., Cho, H. C. \& Marban, E. Wnt signalling suppresses voltage-dependent $\mathrm{Na}(+)$ channel expression in postnatal rat cardiomyocytes. J. Physiol. 593, 1147-1157 (2015).

23. Lu, A. et al. Direct and indirect suppression of Scn5a gene expression mediates cardiac Na+ channel inhibition by Wnt signalling. Can. J. Cardiol. 36, 564-576. https://doi.org/10.1016/j.cjca.2019.09.019 (2020)

24. Lu, A. et al. Inhibition of $\beta$-catenin Increases Voltage-gated Na + Current in Brugada Syndrome Cardiomyocytes (March 30, 2021). Available at SSRN: https://ssrn.com/abstract=3815857 or https://doi.org/10.2139/ssrn.3815857 (2021).

25. Wang, N. et al. Activation of Wnt/beta-catenin signaling by hydrogen peroxide transcriptionally inhibits NaV1.5 expression. Free Radic. Biol. Med. 96, 34-44. https://doi.org/10.1016/j.freeradbiomed.2016.04.003 (2016).

26. Zhao, L., Sun, L., Lu, Y., Li, F. \& Xu, H. A small-molecule LF3 abrogates beta-catenin/TCF4-mediated suppression of NaV1.5 expression in HL-1 cardiomyocytes. J. Mol. Cell Cardiol. 135, 90-96. https://doi.org/10.1016/j.yjmcc.2019.08.007 (2019).

27. Gillers, B. S. et al. Canonical wnt signaling regulates atrioventricular junction programming and electrophysiological properties. Circ. Res. 116, 398-406. https://doi.org/10.1161/CIRCRESAHA.116.304731 (2015).

28. Li, G. et al. Differential Wnt-mediated programming and arrhythmogenesis in right versus left ventricles. J. Mol. Cell Cardiol. 123, 92-107. https://doi.org/10.1016/j.yjmcc.2018.09.002 (2018).

29. Hahn, J. Y. et al. Beta-catenin overexpression reduces myocardial infarct size through differential effects on cardiomyocytes and cardiac fibroblasts. J. Biol. Chem. 281, 30979-30989. https://doi.org/10.1074/jbc.M603916200 (2006). 
30. Oikonomopoulos, A. et al. Wnt signaling exerts an antiproliferative effect on adult cardiac progenitor cells through IGFBP3. Circ. Res. 109, 1363-1374. https://doi.org/10.1161/circresaha.111.250282 (2011).

31. Barandon, L. et al. Reduction of infarct size and prevention of cardiac rupture in transgenic mice overexpressing FrzA. Circulation 108, 2282-2289. https://doi.org/10.1161/01.cir.0000093186.22847.4c (2003).

32. Zelarayán, L. C. et al. Beta-Catenin downregulation attenuates ischemic cardiac remodeling through enhanced resident precursor cell differentiation. Proc. Natl. Acad. Sci. USA 105, 19762-19767. https://doi.org/10.1073/pnas.0808393105 (2008).

33. Gharibeh, L. et al. GATA6 is a regulator of sinus node development and heart rhythm. Proc. Natl. Acad. Sci. USA https://doi.org/ 10.1073/pnas.2007322118 (2021)

34. Cruciat, C. M. \& Niehrs, C. Secreted and transmembrane wnt inhibitors and activators. Cold Spring Harb. Perspect. Biol. 5, a015081. https://doi.org/10.1101/cshperspect.a015081 (2013).

35. Bao, J., Zheng, J. J. \& Wu, D. The structural basis of DKK-mediated inhibition of Wnt/LRP signaling. Science signaling 5, pe22, https://doi.org/10.1126/scisignal.2003028 (2012).

36. Valenta, T., Hausmann, G. \& Basler, K. The many faces and functions of beta-catenin. Embo J. 31, 2714-2736. https://doi.org/10. 1038/emboj.2012.150 (2012).

37. Wang, Z. et al. Wnt signaling activates MFSD2A to suppress vascular endothelial transcytosis and maintain blood-retinal barrier. Sci. Adv. 6, eaba7457, https://doi.org/10.1126/sciadv.aba7457 (2020).

38. Yang, S. \& Huang, X. Y. Ca2+ influx through L-type Ca2+ channels controls the trailing tail contraction in growth factor-induced fibroblast cell migration. J. Biol. Chem. 280, 27130-27137. https://doi.org/10.1074/jbc.M501625200 (2005).

39. Pride, Y. B. et al. Relation between myocardial infarct size and ventricular tachyarrhythmia among patients with preserved left ventricular ejection fraction following fibrinolytic therapy for ST-segment elevation myocardial infarction. Am. J. Cardiol. 104, 475-479. https://doi.org/10.1016/j.amjcard.2009.04.005 (2009).

40. Alexandre, J. et al. Scar extent as a predictive factor of ventricular tachycardia cycle length after myocardial infarction: implications for implantable cardioverter-defibrillator programming optimization. Europace 16, 220-226. https://doi.org/10.1093/europace/ eut289 (2014)

41. Josephson, M. E., Horowitz, L. N. \& Farshidi, A. Continuous local electrical activity. A mechanism of recurrent ventricular tachycardia. Circulation 57, 659-665. https://doi.org/10.1161/01.cir.57.4.659 (1978).

42. Wang, N. C. et al. Clinical implications of QRS duration in patients hospitalized with worsening heart failure and reduced left ventricular ejection fraction. JAMA 299, 2656-2666. https://doi.org/10.1001/jama.299.22.2656 (2008).

43. Dhingra, R. et al. Electrocardiographic QRS duration and the risk of congestive heart failure: The Framingham Heart Study. Hypertension 47, 861-867. https://doi.org/10.1161/01.hyp.0000217141.20163.23 (2006).

44. He, B. J., Boyden, P. \& Scheinman, M. Ventricular arrhythmias involving the His-Purkinje system in the structurally abnormal heart. Pacing Clin. Electrophysiol.: PACE 41, 1051-1059. https://doi.org/10.1111/pace.13465 (2018).

45. Puljevic, D., Smalcelj, A., Durakovic, Z. \& Goldner, V. Effects of postmyocardial infarction scar size, cardiac function, and severity of coronary artery disease on QT interval dispersion as a risk factor for complex ventricular arrhythmia. Pacing Clin. Electrophysiol.: PACE 21, 1508-1516. https://doi.org/10.1111/j.1540-8159.1998.tb00237.x (1998).

46. Deb, A. Cell-cell interaction in the heart via Wnt/ $\beta$-catenin pathway after cardiac injury. Cardiovasc. Res. 102, 214-223. https:// doi.org/10.1093/cvr/cvu054 (2014).

47. Valenta, T., Hausmann, G. \& Basler, K. The many faces and functions of $\beta$-catenin. Embo J. 31, 2714-2736. https://doi.org/10.1038/ emboj.2012.150 (2012).

48. Zuppinger, C., Eppenberger-Eberhardt, M. \& Eppenberger, H. M. N-Cadherin: structure, function and importance in the formation of new intercalated disc-like cell contacts in cardiomyocytes. Heart Fail. Rev. 5, 251-257. https://doi.org/10.1023/a:1009809520 194 (2000).

49. Kuwabara, Y. et al. Increased expression of HCN channels in the ventricular myocardium contributes to enhanced arrhythmicity in mouse failing hearts. J. Am. Heart Assoc. 2, e000150. https://doi.org/10.1161/jaha.113.000150 (2013).

50. Hofmann, F. et al. Ventricular HCN channels decrease the repolarization reserve in the hypertrophic heart. Cardiovasc. Res. 95, 317-326. https://doi.org/10.1093/cvr/cvs184 (2012).

51. Yue, L., Xie, J. \& Nattel, S. Molecular determinants of cardiac fibroblast electrical function and therapeutic implications for atrial fibrillation. Cardiovasc. Res. 89, 744-753. https://doi.org/10.1093/cvr/cvq329 (2011).

52. Percie du Sert, N. et al. The ARRIVE guidelines 2.0: Updated guidelines for reporting animal research. Br. J. Pharmacol. 177, 3617-3624. https://doi.org/10.1111/bph.15193 (2020).

53. Molgat, A. S. et al. Hyperglycemia inhibits cardiac stem cell-mediated cardiac repair and angiogenic capacity. Circulation 130, S70-76. https://doi.org/10.1161/circulationaha.113.007908 (2014).

54. Kapoor, N., Liang, W., Marban, E. \& Cho, H. C. Direct conversion of quiescent cardiomyocytes to pacemaker cells by expression of Tbx18. Nat. Biotechnol. 31, 54-62. https://doi.org/10.1038/nbt.2465 (2013).

55. Liang, W. et al. Role of phosphoinositide 3-kinase \{alpha\}, protein kinase $\mathrm{C}$, and L-type Ca2+ channels in mediating the complex actions of angiotensin II on mouse cardiac contractility. Hypertension 56, 422-429 (2010).

56. Jadhav, A. et al. Catharanthine dilates small mesenteric arteries and decreases heart rate and cardiac contractility by inhibition of voltage-operated calcium channels on vascular smooth muscle cells and cardiomyocytes. J. Pharmacol. Exp. Ther. 345, 383-392. https://doi.org/10.1124/jpet.112.199661 (2013).

\section{Acknowledgements}

This work was supported by the Canadian Institutes of Health Research (Grant number: PJT-148918, to W.L.), McDonald Scholarship and New Investigator Award from Heart and Stroke Foundation of Canada (Grant number: S-17-LI-0866, to W.L.), a Scholarship (to J.W.) and a Postdoctoral Fellowship (to A.L.) from the University of Ottawa Cardiac Endowment Funds at the Heart Institute. Figures 1A,B,E, 5A,B and part of Figure 7A were created with BioRender.com with approved publication licenses.

\section{Author contributions}

J.W. and W.L conceived the experiments. J.W., Y.X., A.L., and H.W. performed experiments. J.W., D.R.D., P.L., R.S.B. and W.L. analyzed and interpreted results and wrote the manuscript. All authors reviewed and approved the manuscript.

\section{Competing interests}

The authors declare no competing interests. 


\section{Additional information}

Supplementary Information The online version contains supplementary material available at https://doi.org/ 10.1038/s41598-021-97176-9.

Correspondence and requests for materials should be addressed to W.L.

Reprints and permissions information is available at www.nature.com/reprints.

Publisher's note Springer Nature remains neutral with regard to jurisdictional claims in published maps and institutional affiliations.

(c) (1) Open Access This article is licensed under a Creative Commons Attribution 4.0 International cc) License, which permits use, sharing, adaptation, distribution and reproduction in any medium or format, as long as you give appropriate credit to the original author(s) and the source, provide a link to the Creative Commons licence, and indicate if changes were made. The images or other third party material in this article are included in the article's Creative Commons licence, unless indicated otherwise in a credit line to the material. If material is not included in the article's Creative Commons licence and your intended use is not permitted by statutory regulation or exceeds the permitted use, you will need to obtain permission directly from the copyright holder. To view a copy of this licence, visit http://creativecommons.org/licenses/by/4.0/.

(C) The Author(s) 2021 